LA W RENCE LIVERMORE NATIONAL LABORATORY

\title{
Edge Simulation Laboratory Project Report
}

R. H. Cohen, M. Dorf, M. Dorr, T. D. Rognlien

February 25, 2011 
This document was prepared as an account of work sponsored by an agency of the United States government. Neither the United States government nor Lawrence Livermore National Security, LLC, nor any of their employees makes any warranty, expressed or implied, or assumes any legal liability or responsibility for the accuracy, completeness, or usefulness of any information, apparatus, product, or process disclosed, or represents that its use would not infringe privately owned rights. Reference herein to any specific commercial product, process, or service by trade name, trademark, manufacturer, or otherwise does not necessarily constitute or imply its endorsement, recommendation, or favoring by the United States government or Lawrence Livermore National Security, LLC. The views and opinions of authors expressed herein do not necessarily state or reflect those of the United States government or Lawrence Livermore National Security, LLC, and shall not be used for advertising or product endorsement purposes.

This work performed under the auspices of the U.S. Department of Energy by Lawrence Livermore National Laboratory under Contract DE-AC52-07NA27344. 


\section{EDGE SIMULATION LABORATORY PROJECT REPORT, CY 2010}

In 2010 The Edge Simulation Laboratory (ESL) embarked upon the plan laid out in the renewal proposal submitted in December 2009. This proposal called for initially parallel efforts addressing the physics of the closed-flux-surface pedestal region, using existing computational tools (GYRO, BOUT++) and analytic modeling, and physics of the scrape-off layer via development of the new edge gyrokinetic code COGENT. Progress in the former area is described in a series of monthly progress reports prepared by General Atomics; these are attached as a set of appendices (describing work done in the month prior to the indicated date). Progress in the latter area, as well as associated theoretical development, is described below.

\section{COGENT CODE DEVELOPMENT AND VERIFICATION ACTIVITIES}

COGENT is a $4^{\text {th }}$-order finite-volume continuum code being developed collaboratively by a math team at LLNL and LBNL and a physics team based at LLNL (with participation from also from UCSD). In 2010 the principal progress areas were improvements in numerics and addition of collision models to the existing closed-flux-surface ("core", or "core-plus-limiter" model), validation activities including geodesic-acoustic mode (GAM) simulations and collisional relaxation and neoclassical tests, and implementation of ingredients necessary for the extension of the code to true divertor geometry.

\section{GEODESIC ACOUSTIC MODE (GAM) SIMULATIONS}

In late 2009 we began a campaign with COGENT to verify the code by performing simulations of geodesic acoustic modes (GAMs) and comparing with other codes and theory. The intention was to perform simulations with the same parameters as used in the multi-code comparison of Xu et al. [1]. As of the end of 2009 we had first simulations in hand, but comparison was limited by numerical issues which interfered with finding a converged, accurate potential, by some incorrect understanding of the assumptions and parameters in some of the comparison simulations, and by lack of an in-house capability to evaluate the theory of Gao et al. [2].

During 2010, the numerical issues were resolved by a more numerically appropriate formulation of the coefficient of the Boltzmnan electron model, by resolving issues associated with fourthorder accurate products of quantities, and by finding some bugs, while the understanding of assumptions and parameters in comparison simulations was resolved by discussion with authors.

The development of an in-house capability to evaluate the theory of Gao entailed developing an accurate procedure for numerical solution to Gao's dispersion relation,

$$
\left[1+\frac{T_{i}}{T_{e}}\left(1-\delta_{L, 0}\right)\right] \varphi_{L}=\int_{C_{L}} \frac{d^{3} v e^{-v^{2}}}{\pi^{3 / 2}} J_{0}^{2}\left(k v_{\perp}\right) \sum_{n, l=-\infty}^{\infty} \varphi_{l} i^{-l+L} J_{n}(x) J_{n-l+L}(x) \frac{\xi}{\xi+(n-l) v_{\|}}
$$

with $x=k q\left(2 v_{\|}{ }^{2}+v_{\perp}^{2}\right) / 2 v_{\|}, \xi=q R \omega / v_{t i}, k=k_{\perp} v_{t i} / \omega, v_{t i}=\left(w T_{i} / m_{i}\right)^{1 / 2}$, and $\mathrm{v}$ is normalized to $v_{t i}$. The procedure entailed development of an asymptotic analysis for small $x$ that takes into account correct Landau contours and determination of the coefficients $a_{j}$ in the Bessel function expansion 


$$
\sum_{n=-\infty}^{\infty} n^{k} J_{n}(x) J_{n+m}(x) \equiv \sum_{j=0}^{k} a_{j} x^{j}
$$

The resulting procedure was applied to evaluation of the dispersion relationship for $\tau=T_{e} / T_{i}=0$ and 1 and compared with the simulation results. As shown in Fig 1, excellent agreement is found for the GAM decay rate between COGENT and analytic theory. For $\tau=0$ there is comparably good agreements with XGC-1; the tests of the latter cod keep only the flux-surfaceaveraged component of the potential only and so cannot be applied to $\tau=1$. The residual differences with GYRO may be due to a different way of measuring decay rates. (The decay rate

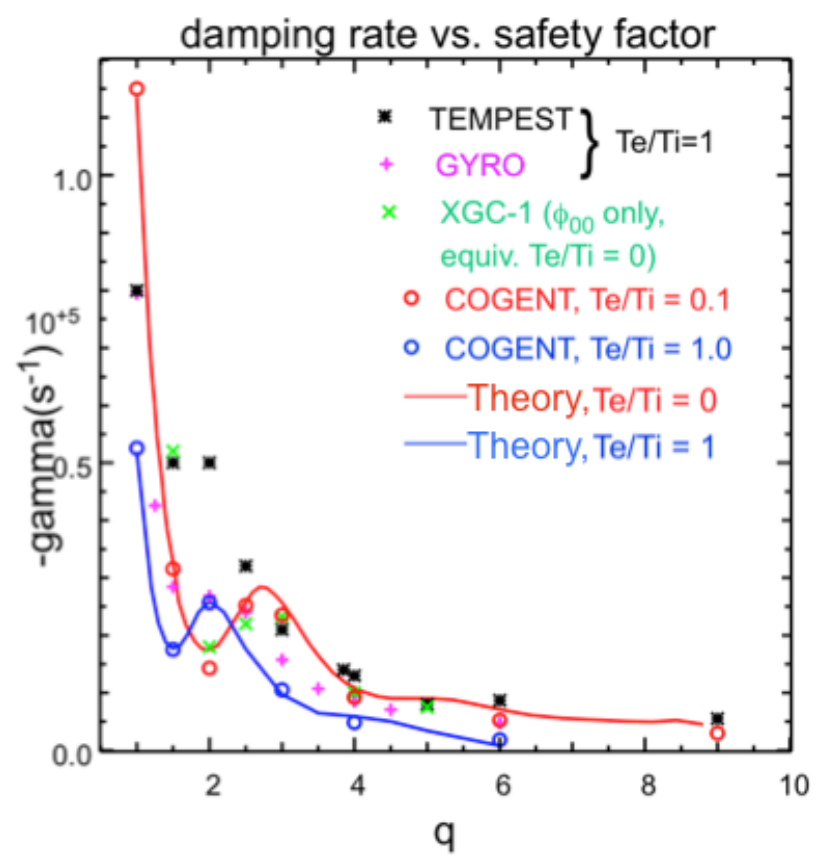

Fig 1. Comparison of GAM damping rates from COGENT and other codes with Gao theory

for COGENT results is obtained by determining the value of $\gamma$ such that $\exp (\gamma t) \phi(t)$ is approximately constant for some period of time following a short initial transient.) Excellent agreement is also found for the real frequency. Comparison of the change of decay rate between with several refinements of grid resolution demonstrates clear fourth-order convergence, as expected for COGENT's discretizations. These results were presented in Ref. 3.

\section{Impementation of Collision Models in COGENT}

Several model collision operators have been implemented and tested in the COGENT. These include a model "parallel drag-diffusion" collision operator, the Lorentz operator, and the linearized Fokker-Plank collision operator in the form proposed by Abel et al in Ref. [4].In addition, the analytical formulation of nonlinear isotropic model for collisions has been developed. In what follows, a brief description of collision models along with the summary of initial tests is presented. 
(a) "Parallel drag-diffusion" collision operator

A model "parallel drag-diffusion" operator,

$$
C_{\|}[f]=v_{\text {coll }} \frac{\partial}{\partial v_{\|}}\left(v_{\|} f+\frac{T}{m} \frac{\partial f}{\partial v_{\|}}\right),
$$

provides drag and diffusion in the parallel velocity space. Here, $v_{c o l l}$ is the collision frequency, and $m$ and $T$ correspond to the species mass and equilibrium temperature, respectively. The implementation of this collision model has been tested for the Pastukhov-type problem, where particles confined along the magnetic field direction by a square electrostatic potential well become untrapped due to the re-population of the high-parallel-velocity distribution function by

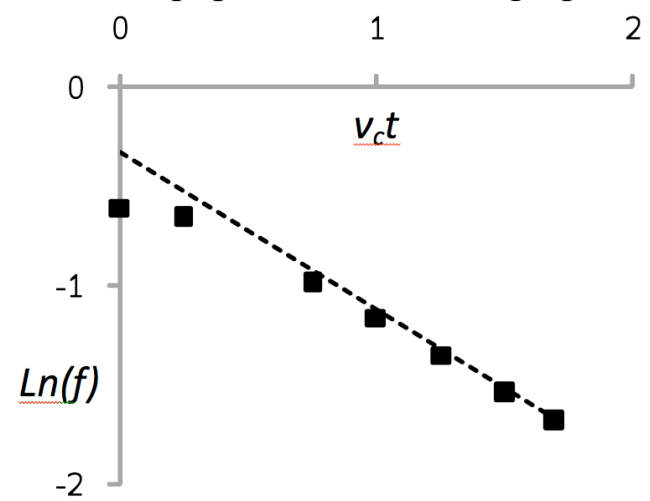

Fig.2. COGENT simulations (dots) of the decay of $\mathrm{f}$ versus time in an electrostatic well with the particle collision model of Eq. (1). The asymptotic decay obtained analytically is given by the dashed line. Here, a fitting parameter is used to locate the vertical position of the dashed line.

collisions. Excellent agreement with an analytical theory was observed, and the results are presented in Fig.2. The figure shows the decay of particle distribution function $f$ due to the parallel loss over a potential barrier. The results correspond to the case of weak collisions, where the collision frequency is much less than the parallel bounce frequency.

(b) Lorentz collision operator

The Lorentz operator,

$$
C_{L}[f(\xi, v)]=v_{\text {coll }} \frac{1}{2} \frac{\partial}{\partial \xi}\left(\left(1-\xi^{2}\right) \frac{\partial f}{\partial \xi}\right),
$$

provides pitch angle scattering in the velocity space. Here, $v_{\text {coll }}$ is the collision frequency, $\xi=v_{\|} / v$ and $v$ are the particle pitch-angle variable and velocity, and a gyrophase-independent distribution function is assumed. Note that although the Lorentz operator conserves particles energy analytically, spurious diffusion in the energy space appears (Fig. 2) due to approximate (finitedifference) numerical evaluation of the operator using the parallel velocity $v_{\|}$and magnetic moment $\mu$ coordinates. Therefore, it is of particular importance to develop and implement a higher-order finite-difference scheme that can minimize the effects of spurious energy diffusion. Figure 3 demonstrates that the spurious diffusion is significantly suppressed (to a tolerable level) for the case where the 4 -th order accurate numerical scheme is implemented.

In order to test the performance of the implemented Lorentz operator, full-f simulations of neoclassical transport have been performed. The results are found in very good agreement with the analytical theory developed in Ref. [5] (see Fig.4). 

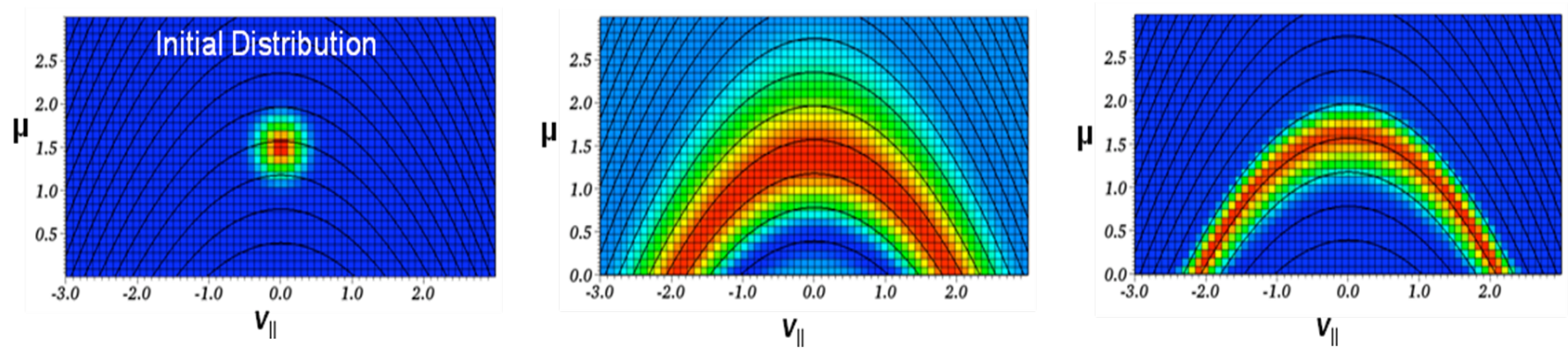

Fig.3. COGENT simulations of the pitch-angle scattering of an initial distribution function represented by a blob in the velocity space. The figure shows plots of (a) the initial distribution $(t=0)$, (b) distribution function at $\mathrm{t}=4 / \mathrm{v}_{\text {coll }}$ obtained making use of the second-order accurate implementation of the Lorentz operator, and (c) distribution function at $\mathrm{t}=4 / \mathrm{v}_{\text {coll }}$ obtained makin use of the fourth-order accurate implementation of the Lorentz operator. The solid black curves illustrate the contours of a constant energy. Note that different scales in color schemes are used in the frames, and the maximum values of the distribution function in frames (a), (b), and (c) are related as 1:0.13:0.067, correspondingly.

\section{(c) Linearized model Fokker-Plank collision operator}

For the case where a distribution function is close to a Maxwellian distribution, i.e. $f=F_{m}+\delta f$, $\delta f<<F_{m}$, an accurate collision model can be provided by a linearized model Landau collision operator. A general form of a like-particle (inner-species) linearized collision operator is given by

$$
C[f, f] \cong C\left[\delta f, F_{M}\right]+C\left[F_{M}, \delta f\right],
$$

where $>$ is the full Landau operator, the "test-particle" term $C\left[\delta f, F_{M}\right]$ describes collisions with a Mawellian background, and $C\left[F_{M}, \delta f\right]$ is customary referred to as the "field-particle" term. The "test-particle" term can be evaluated analytically, and for the case where the distribution function is gyrophase-independent is given by
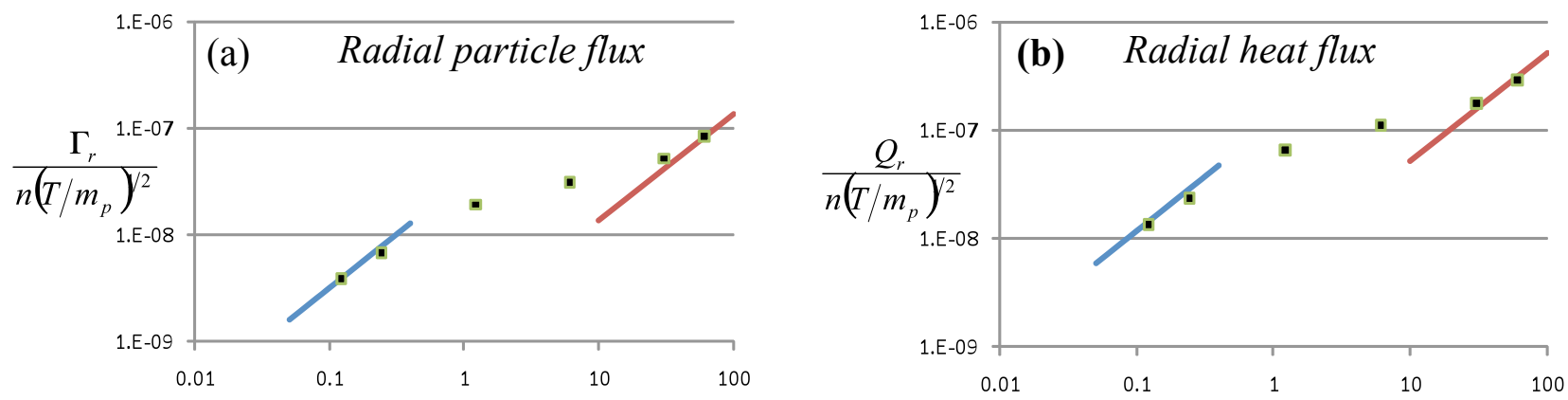

Fig.4. COGENT simulations (dots) of the radial neoclassical ion transport for the case of the Lorentz collision operator. Shown the plots of (a) particle flux, and (b) heat flux versus the normalized collision frequency $v^{*}=\varepsilon^{-3 / 2} v \sqrt{2} q R_{0} / v_{t h}$. The red and blue lines correspond to the analytical calculations [5] in the banana and the Pfirsch-Schluter regimes, respectively. The parameters of the simulation correspond to safety factor $q=3$, inverse aspect ratio $\varepsilon=0.1$, ion temperature $T=3 \mathrm{KeV}$, ion mass $m_{i}=2 m_{p}$, major radius $R_{0}=45.6 \mathrm{~m}$, toroidal magnetic field on axis $B_{0}=7.5 \mathrm{~T}$, inverse temperature and density gradients $\kappa_{n}=$ $\kappa_{T}=10 / R_{0}$, and the magnetic flux tubes have nested circular cross-sections. 


$$
C[\delta f, f]=\frac{v_{D}(v)}{2} \frac{\partial}{\partial \xi}\left(1-\xi^{2}\right) \frac{\partial \delta f}{\partial \xi}+\frac{1}{v^{2}} \frac{\partial}{\partial v}\left[\frac{v_{s}(v)}{2} v^{3} \delta f+\frac{v_{\|}(v)}{2} v^{4} \frac{\partial \delta f}{\partial v}\right],
$$

where the definition of the transport coefficients in Eq. (4), i.e., $v_{D}, v_{s}$, and $v_{\|}$, can be found in [4]. Note that the test-particle term includes both pitch-angle scattering and energy diffusion. The exact evaluation of the "field-particle" term however still requires intensive numerical calculation of the Rosenbluth potentials, and therefore it is typically modeled by "restoring" terms, which provide conservation properties of the full linearized collision operator. The implementation of the linearized collision operator in COGENT makes use of the model fieldparticle term proposed by Abel, et al [4],

$$
C\left[F_{M}, \delta f\right]=\left\{v_{s}(v) \frac{2 v_{\|} U[\delta f]}{v_{t h}^{2}}+v_{E}(v) \frac{v^{2}}{v_{t h}^{2}} Q[\delta f]\right\} F_{M} .
$$

Here, $\quad U[\delta f]=(3 / 2) \int v_{s} v_{\|} \delta f d^{3} v / \int\left(v / v_{t h}\right)^{2} v_{s} f_{M} d^{3} v, \quad Q[\delta f]=\int v_{E} v^{2} \delta f d^{3} v / \int\left(v / v_{t h}\right)^{2} v v^{2} f_{M} d^{3} v$, $v_{E}=-\left(1 / v^{4} F_{M}\right) \partial\left(v^{5} v_{\|} F_{M}\right) / \partial v$, and a gyrophase-independent distribution function is assumed. The field-particle term [Eq. (5)] provides conservation of particles, the parallel momentum and energy of the distribution; obeys H-theorem, vanishes on a perturbed Maxwellian, efficiently dissipates small-scale structure in the velocity space; and can be conveniently generalized for gyrokinetic simulations taking into account finite Larmor radius (FLR) effects [4].

The fourth-order accurate numerical implementation of the linearized collision operator given by Eq. (3)-(5) has been recently accomplished in COGENT. The initial successful tests of the testparticle collision term include the analysis of the parallel momentum relaxation (for the case of a non-zero initial parallel velocity), and the thermal relaxation (for the case where the temperature of the particle distribution is different from the temperature of a Maxwellian background). Furthermore, the conservative properties of the full linearized like-particle collision operator (including both test-particle and field-particle terms) is observed.

\section{(d) Nonlinear isotropic model for collisions}

A linearized collision operator becomes of limited validity in a tokamak edge region where a particle distribution function can be substantially different from a Maxwellian distribution. For instance, non-thermal tails may develop due to the fact that the mean free path for the suprathermal particles can become comparable to a connection length in the scrape-of-layer (SOL) region. Furthermore, anisotropy in the distribution function in the edge region can develop due to the presence of particle loss regions the particle phase-space.

A simple nonlinear collision model taking into account isotropic nonthermal effects has been formulated by straightforward generalization of the linearized model described in the previous section. The nonlinear model operator for like-particle collisions has the form

$$
C[f, f] \rightarrow C[f,\langle f\rangle]+P[f](\mathbf{v})\langle f\rangle,
$$

where $\langle f\rangle \equiv \frac{1}{2} \int \sin \xi d \xi f$ is the isotropic part of the distribution function (a gyrophaseindependent distribution function is assumed). Note that the first term on the right hand side describes collisions with the isotropic nonthermal background, and has the form of Eq. (4), with the transport coefficient now expressed in terms of 1D velocity integrals over the isotropic 
background. The field-particle term (second term on the right-hand-side) is obtained by straightforward generalization of the analysis in Ref. [5], and is given by

$$
P[f]=2 \bar{v}_{s}(v) v_{\|} U[\delta f]+\bar{v}_{E}(v) v^{2} Q[\delta f]+\left(A v^{2}+B\right) .
$$

For the case of a Maxwellian background it reduces to the field-particle term given in Eq. (5), i.e. $A=B=0, \bar{v}_{s}=v_{s}, \bar{v}_{E}=v_{E}, \bar{U}=U, \bar{Q}=Q$. It should be noted that a similar nonlinear model for the test-particle collision term was implemented in the particle-in-cell PIC simulations performed in Ref. [6], however a different method (based on the Langeven-equation approach to collisions) was used to restore the parallel momentum and energy of the particle distribution.

\section{Incorporation of radial diffusion model}

We are in the process of incorporating into COGENT a radial diffusion operator to model the effects of anomalous transport in the $4 \mathrm{D}$ version of the code. This is following on similar work done in the predecessor code TEMPEST, in which a diffusion operator with a velocity-dependent diffusion coefficient, in combination with an advection operator, was devised to allow reproduction in a kinetic code of the effects of an arbitrary diagonal transport matrix in a fluid code, when applied to a Maxwellian distribution function. The goal here is to provide a "kinetic UEDGE", and also to provide the basis for eventual coupling of 4D (transport) and 5D (turbulence) versions of the kinetic code. A simple form of the diffusion operator is now operational in the code and passing verification tests (comparison with analytic solutions for pure diffusion); extension of the implementation is now in progress. The current work, like the preceding TEMEPST work, is being carried out by a graduate student at UCSD with coaching from LLNL staff and UCSD faculty.

\section{Numerical/algorithmic advances}

The gyrokinetic Poisson equation with Boltzmann electrons constitutes a nonlinear equation to be solved for the potential. Using a standard Newton iteration, a Jacobian system must be solved at least approximately in each iteration. The Jacobian is the sum of the linear gyrokinetic Poisson operator and a contribution from the Boltzmann relation. Ordinarily, such Poisson-Boltzmann operators are quite easy to invert, since the Boltzmann relation adds an additional positive symmetric term to an already positive symmetric operator. In this case, however, the dependence of the electron-density prefactor $n_{0}\left[n_{e}=n_{0} \exp \left(e \phi / T_{e}\right)\right]$ on the potential $\left[n_{0}=\left\langle n_{i}\right\rangle /\left\langle\exp \left(e \phi / T_{e}\right)\right\rangle\right.$ where \langle\rangle denotes a flux-surface average in the denominator and either a flux-surface or global average in the numerator)] is such that the contribution to the Jacobian from the Boltzmann relation is nonsymmetric. A more serious issue is the fact that for for the typical situation where radial scale lengths are many gyroradii, the Jacobian matrix has a near null space comprised of vectors constant along flux surfaces. We therefore developed a strategy for removing the null space component via a tridiagonal solve in the radial direction, combined with a BiCGStab (biconjugate gradient stabilized) algorithm to obtain the solution component in the null space complement, with multigrid as a preconditioner.

We also made refinements to the treatment of fourth-order averages of products of vector quantities that better accounted for the strong asymmetry of directions along and across magnetic field lines. These refinements, in combination with the improved treatment of the gyrokinetic Poisson-Boltzmann equation described above, enabled the successful GAM tests described 
above, in particular enabling good convergence of the field solve, the favorable comparison with analytic theory, and an explicit demonstration of fourth-order convergence of the GAM simulations with respect to mesh resolution. In particular, the fourth-order accuracy of the GAM results was confirmed via Richardson extrapolation using a sequence of grids successively refined in all phase space directions. In Figure 5, the differences $e_{N} \equiv\left\|n_{N}-n_{4}\right\|$ are plotted for 1 $\leq N \leq 3$, approximating the error in the solutions on the first three refined grids by assuming that the solution on the fourth refined grid is exact. The black curve (only whose slope and not vertical position is relevant) indicates fourth-order convergence. These tests were described in Refs. [7] and [3].

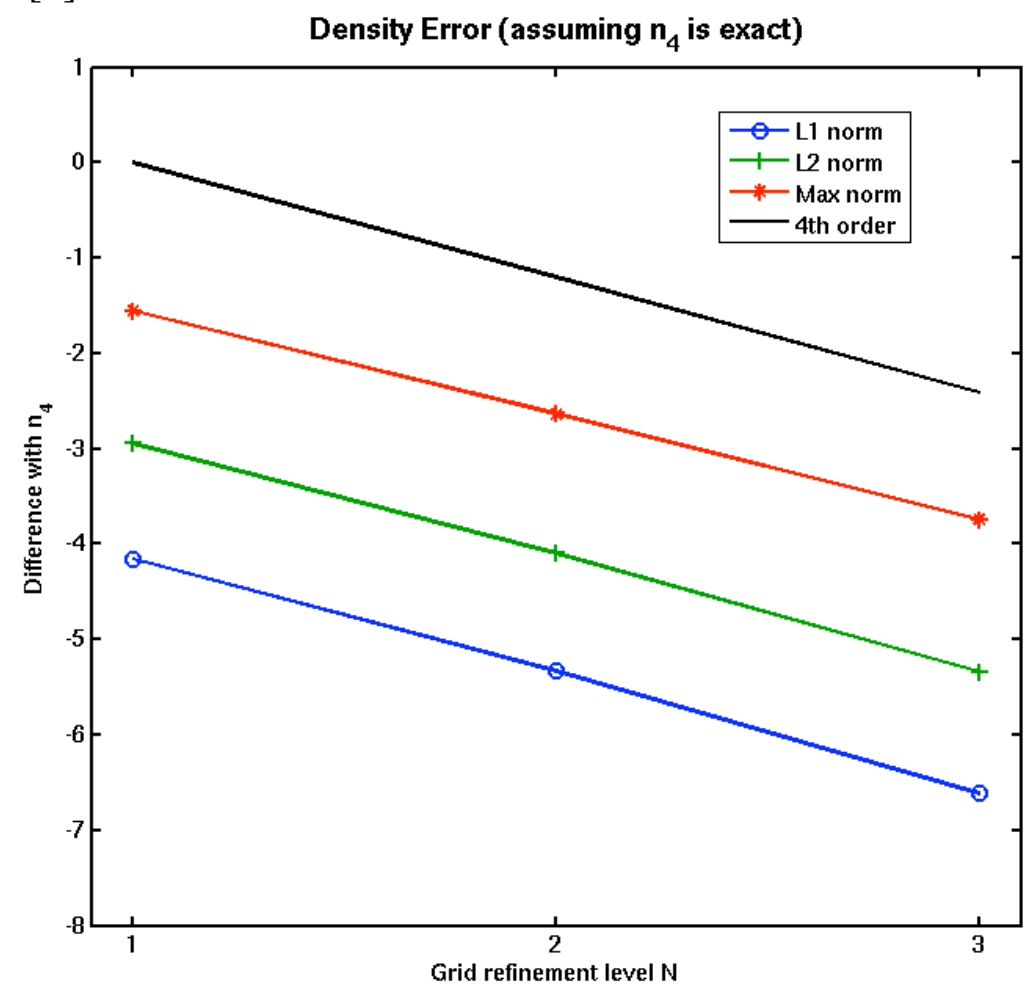

Fig. 5. Error as a function of refinement level, assuming the solution on the finest grid is exact. The black curve indicates a fourth-order convergence rate.

\section{Progress toward a divertor version of the code}

A key ingredient in our strategy for deploying a divertor-geometry version of COGENT is the exploitation of mapped multiblock technology, whereby the closed-flux-surface edge, scrape-offlayer, and private-flux regions of a tokamak are described by separate blocks of grids which communicate through their common boundaries. This functionality was recently released in CHOMBO, and we are in the process of implementing this technology in COGENT, revamping data structures and generalizing interfaces as needed. We have also been developing the software necessary to provide the needed metric coefficients for numerically prescribed divertor configurations and extending the geometry classes as needed to handle the increased geometric complexity; this included developing interpolation and smoothing algorithms that correctly capture the effects of the separatrix $X$ point to the requisite $\left(4^{\text {th }}\right)$ order. We anticipate a 
functioning advection code this spring and intend to have a fully functional code (including field solve and at least some colllisional capability) by the end of FY 2011.

\section{Development of associated theory}

We have been working over the past three years on a formal development of gyrokinetic equations in orderings that are more appropriate for the plasma edge than the traditional equation sets. Our progress in the past year has included the following: Tthe gyrokinetic minimal models (both canonical and symplectic versions) were recast in a more systematic way as reductions of the full gyrokinetic equations under a specific ordering on the electromagnetic perturbations. We extended Dimits-LoDestro-Dubin $1992\left(\mathrm{~V}_{\mathrm{ExB}}<<\mathrm{V}_{\text {th }}\right)$ ordering gyrokinetic derivation to toroidal geometry. This is particularly useful in that the resulting equations are essentially the same as previous sets derived under more restrictive standard orderings, and a numerical implementation path for these is already in place. And, we extended large-flow $\left[\mathrm{V}_{\mathrm{ExB}} / \mathrm{V}_{\text {th }}=\mathrm{O}(1)\right]$ ordering gyrokinetic derivation to toroidal geometry and electromagnetic perturbations. This work was presented in invited talks at the 2010 Sherwood Fusion Theory Conference and at the Cambridge UK Workshop on Gyrokinetics in Laboratory and Astrophysical Plasmas, (August 10, 2010).

\section{Acknowledgment}

ESL work is performed under the auspices of the U.S. Department of Energy under contracts DE-AC52-07NA27344 at Lawrence Livermore National Laboratory and DE-AC02-05CH11231 at Lawrence Berkeley National Laboratory.

\section{References:}

[1] X.Q. Xu, K. Bodi, J. Candy et al., Nucl. Fusion 49, 065203 (2009).

[2] Xhe Gao, K. Itoh, H. Sanuki, and J.Q. Dong, Phys. Plasmas 15, 072511 (2008)

[3] T.D. Rognlien, K. Bodi, R.H. Cohen et al., "Advances in understanding tokamak edge/scrape-off layer transport", In Proceedings of the IAEA Fusion Energy Conference, Daejeon, South Korea, October 2010.

[4] I. G. Abel, M. Barnes, S. C. Cowley, W. Dorland, and A. A. Schekochihin, Phys. Plasmas, 15, 122509 (2008).

[5] Z. Lin, W. M. Tang, and W. W. Lee, Phys. Plasmas 2, 2975 (1995).

[6] W. M. Manheimer, M. Lampe, and G. Joyce, J. Comput. Phys. 138, 563 (1997).

[7] M. R. Dorr, R. H. Cohen, P. Colella, M. A. Dorf, J. A. F. Hittinger, and D. F. Martin. Numerical simulation of phase space advection in gyrokinetic models of fusion plasma. In Proceedings of SciDAC 2010, 2010. 


\title{
ESL/GA Progress Report
}

\author{
E. A. Belli, J. Candy, P. B. Snyder
}

February 2010

NEO has been used to analyze the deuterium ion flow of DIII-D L-mode plasmas in the edge. In these studies, a new method has been adopted to determine the shift in the parallel flow due to the radial electric field. The equilibrium-scale radial electric field, which is an input in NEO, is usually determined via the pressure balance equation, using carbon impurity toroidal and poloidal flow measurements along with the measured radial pressure gradients. However, the carbon poloidal flow measurements often have large uncertainties and are usually neglected from this equation under the assumption that the poloidal flow term is small compared to the toroidal flow term. This is generally true in the core but not in the edge. Thus, for these studies, in which we are interested in comparing the deuterium ion parallel flows, we have used the carbon toroidal flow measurements as a calibration to determine the radial electric field by forcing the NEO value to match the measured value at $\theta=0$. This recalibration method is valid only the weak rotation limit, since then the neoclassical flow coefficient does not depend on the radial electric field. The results are shown in figure 1. The measurements, which are not shown in this figure, were done outside the separatrix. Initial comparisons find that the NEO results and the measurements qualitatively approach each other as $\rho_{N} \rightarrow 1$, indicating that the flow remains essentially neoclassically-driven even close to the plasma edge. In figure 1, we confirm that the component of the ion parallel flow due to the radial electric field, $u_{\|, E r}=-c I /\left(\psi^{\prime} B\right) \partial \Phi / \partial r$, is dominant compared to the component due to the pressure gradients, which includes the neoclassical flow coefficients, $u_{\|, p}=c T_{0 a} I /\left(z_{a} e \psi^{\prime} B\right)\left[1 / L_{n a}+\left(1-k_{a}\right) / L_{T a}\right]$, until very close to the edge and thus we not just comparing the calibrated components in the experimental comparison. 

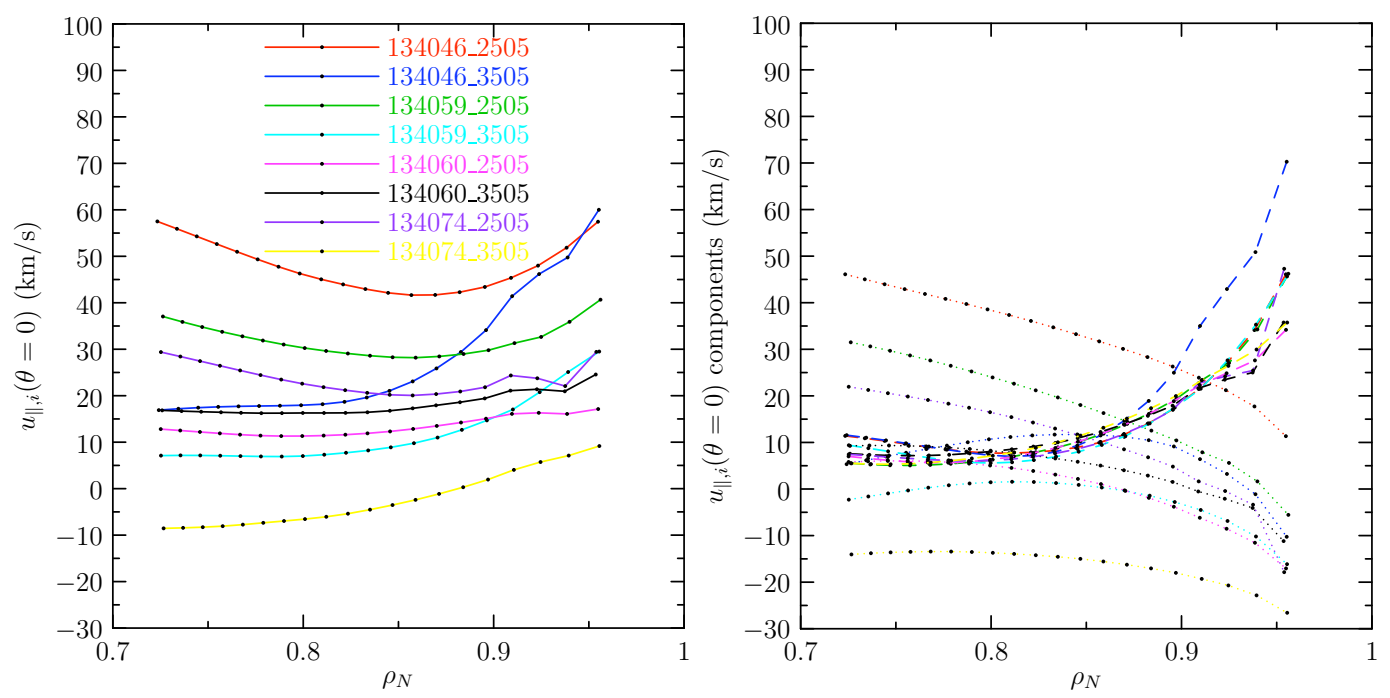

Figure 1: Radial profiles of the deuterium ion parallel flow from NEO for various DIII-D L-mode plasmas. The figure on the left shows the total flow, while the figure on the right shows the components due to the pressure gradients (dashed lines) and the components due to the radial electric field shift (dotted lines).

GYRO simulations of NSTX plasmas have found that finite $\beta^{\prime}$ can produce a hybrid ITG-KBM mode. This is shown in figure 2 . Here we have used the new GYRO eigenvalue solver. In these studies, we have varied $\beta$ at fixed values of $\beta^{\prime}$. All of the results include the full electromagnetic perturbations $\left(\delta A_{\|}\right.$and $\left.\delta B_{\|}\right)$. For low values of $\beta^{\prime}$, we find a distinct dominant ITG mode at low $\beta$ and a distinct dominant $\mathrm{KBM}$ at high $\beta$. As $\beta^{\prime}$ increases from zero, the ITG mode becomes more unstable while the KBM becomes more stable. When $\beta^{\prime}$ reaches $1 / 10$ of the actual experimental value, we see that a cusp forms in the linear growth rate plot just below $\beta_{e}=\beta_{e, \exp }$. The real frequency plot shows that here the ITG and KBM branches have merged to form a continuous mode. For the hybrid mode, at large $\beta, \delta A_{\|}$exhibits the KBM-like symmetry, in which the real and imaginary components are out-of-phase with each other. As $\beta$ decreases, the imaginary component becomes smaller more quickly and eventually reverses to become in-phase with the real component, which is the ITG-like symmetry. 

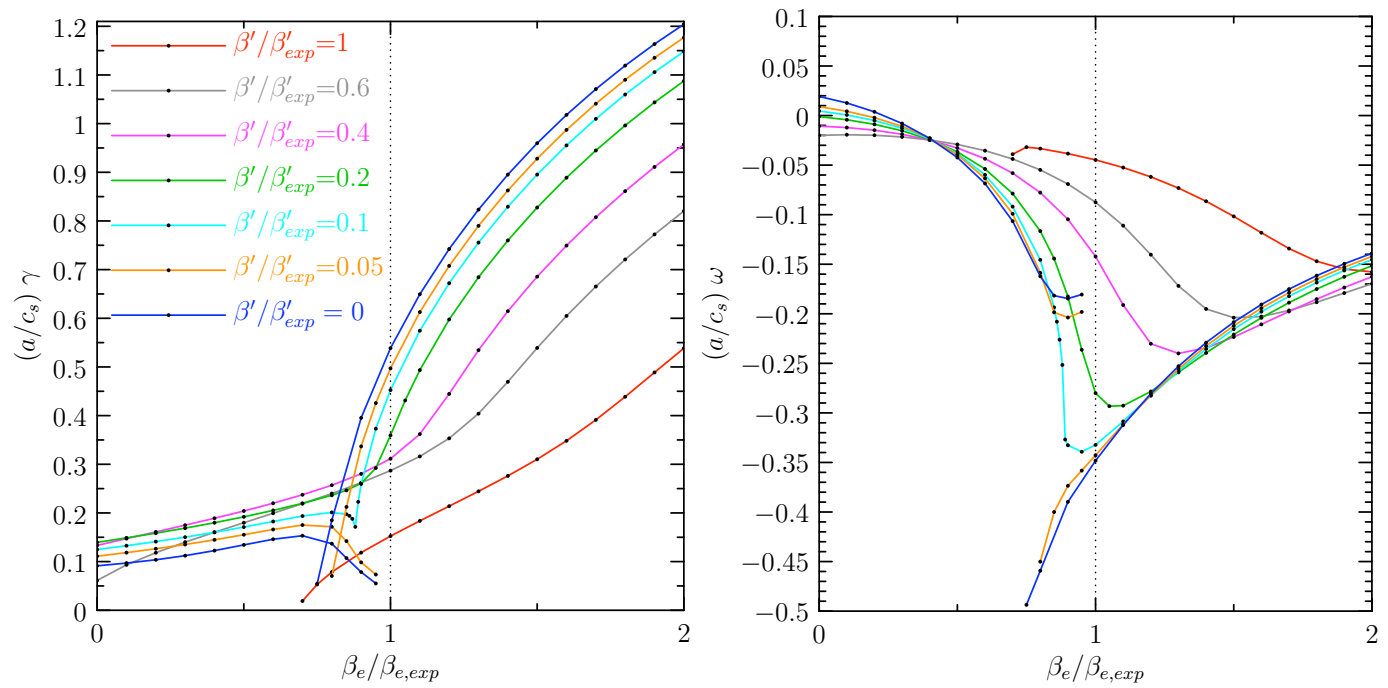

Figure 2: Linear growth rate and real frequency vs. the electron beta $\left(\beta_{e}=\right.$ $\left.8 \pi n_{0 e} T_{0 e} / B_{u n i t}^{2}\right)$ relative to the actual experimental value at various fixed values of the $\beta$ radial gradient relative to the actual experimental value. The simulation parameters are based on NSTX discharge \#132641 at $k_{\theta} \rho_{s}=$ 0.25 . 


\title{
ESL/GA Progress Report
}

\author{
E. A. Belli, J. Candy, P. B. Snyder
}

April 2010

A new tool, VGEN, is being developed to compute the radial electric field based on NEO for input to GYRO, TGYRO, and subsequent NEO calculations. For GYRO and NEO, the source of the input $E_{r}$ is often arbitrary, i.e. using some combination of measured flows, computed flows, and analytic theory, since the measured impurity poloidal rotation data is often either unavailable or has large uncertainties. The new tool takes as input either both the measured impurity toroidal and poloidal velocity profile data or just the measured impurity toroidal velocity profile data. Given both profiles, $E_{r}$ is computed from force balance,

$$
E_{r}=\frac{1}{n_{a} e_{a}}|\nabla r| \frac{\partial p}{\partial r}+v_{\varphi} B_{\theta}-v_{\theta} B_{\varphi}
$$

in a manner that is consistent with the GYRO/NEO geometry implementation. Given only the toroidal velocity, $E_{r}$ is computed by matching the NEO value with the measured value at the outboard midplane. This was tested previously in the weak rotation limit in DIII-D L-mode deuterium flow studies (see our Feb. 2010 report). In this limit, the neoclassical flow coefficient is independent of $E_{r}$ and thus,

$$
-c \frac{d \Phi}{d \psi}=\frac{v_{\varphi, \text { measured }}^{(i m p)}-v_{\varphi, \text { neo }}^{(i m p)}\left(E_{r}=0\right)}{R} .
$$

In the strong rotation limit, the problem of $v_{\varphi, \text { measured }}^{(i m p)}=v_{\varphi, \text { neo }}^{(i m p)}$ becomes an expensive nonlinear root finding problem, which we solve using Newton's method. In each case, the corresponding NEO-computed flows for all species, assuming either weak or strong rotation as specified, are also given as output. VGEN is implemented using the NEO subroutine interface, which itself has 
recently been upgraded to allow for general Grad-Shafranov equilibrium geometry in radially local mode. Comparisons of the three methods are in progress. Preliminary results using data from DIII-D L-mode discharges indicate that the finite-Mach number corrections are small. Small differences are also found between the VGEN force balance $E_{r}$ and the previouslycomputed force balance $E_{r}$ near the magnetic axis and near the plasma edge, most likely due to geometry assumptions. 


\title{
ESL/GA Progress Report
}

\author{
E. A. Belli, J. Candy, P. B. Snyder
}

May 2010

NEO has been upgraded to account for the direction of the magnetic field. Two input parameters, ip_ccw and bt_ccw have been introduced to specify the orientation of the plasma current and toroidal magnetic field. While this does not affect the particle and energy fluxes, it does affect the flows and momentum flux.

Studies of the accuracy of the new NEO-based tool VGEN, which computes the flows and radial electric field, are on-going. Results for a typical DIII-D L-mode shot are shown in figure 1 . Here we find that $E_{r}$ is mainly determined by the toroidal flow component. The component of $E_{r}$ due to the poloidal flow is small but not negligible in the core. In the edge, the poloidal and toroidal flow components are comparable. Small differences are seen between a method which uses force balance but neglects $v_{\theta}$ and the new method which computes $E_{r}$ by forcing the NEO-computed $v_{\varphi}$ to match the measured value. It is not yet clear why a larger improvement was not seen with the latter method. Only slight differences were found by including finite Mach number effects. The effect of finite Mach number on both the deuterium and carbon flows is negligible, as shown in figure 2. In this figure, it is clear from the deviation between the measured and NEO-computed values that the poloidal flow is not purely driven by neoclassical processes for this case. More cases are needed to fully study the approaches.

Studies of the effects of compressional magnetic perturbations on the linear gyrokinetic stability of unusually high-beta DIII-D H-mode plasmas (which operate within $10 \%$ of the high-n ideal beta limit) using the new GYRO eigenvalue solver find a non-negligible destabilizing effect at low $k_{\theta} \rho_{s}$ in the core $(\mathrm{r} / \mathrm{a}=0.6)$. Unlike our previously-studied NSTX case, here we 

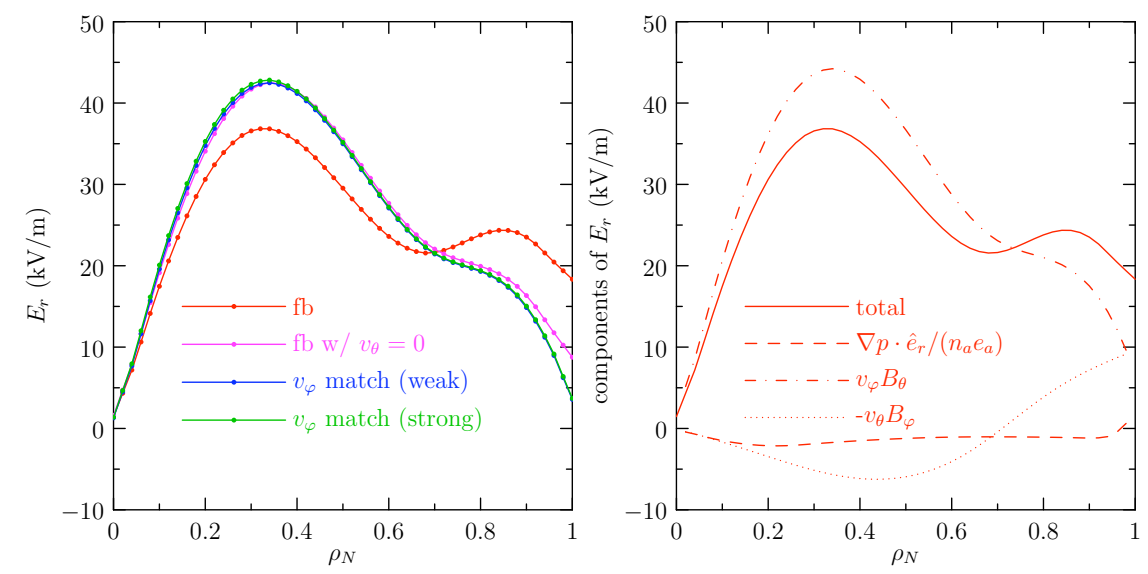

Figure 1: The radial electric field profile for DIII-D L-mode shot \#101391 computed using VGEN, given as input the measured poloidal and toroidal carbon flows, in addition to the measured equilibrium profile data. Here we compare the (exact) force-balance calculation ("fb") with various approximations which neglect the poloidal flow data. The curves labeled " $v_{\varphi}$ match" compute $E_{r}$ by matching the NEO and measured carbon toroidal flow at the outboard midplane, assuming either the weak or strong rotation limit.

find that the dominant mode at the experimental $\beta$ is an ITG mode, rather than a hybrid ITG/KBM mode. Little effect was seen for the higk-k ETG mode. Extensions to the edge are in progress. Preliminary results at $\mathrm{r} / \mathrm{a}=0.9$ find a similar effect at low $k_{\theta} \rho_{s}$. 

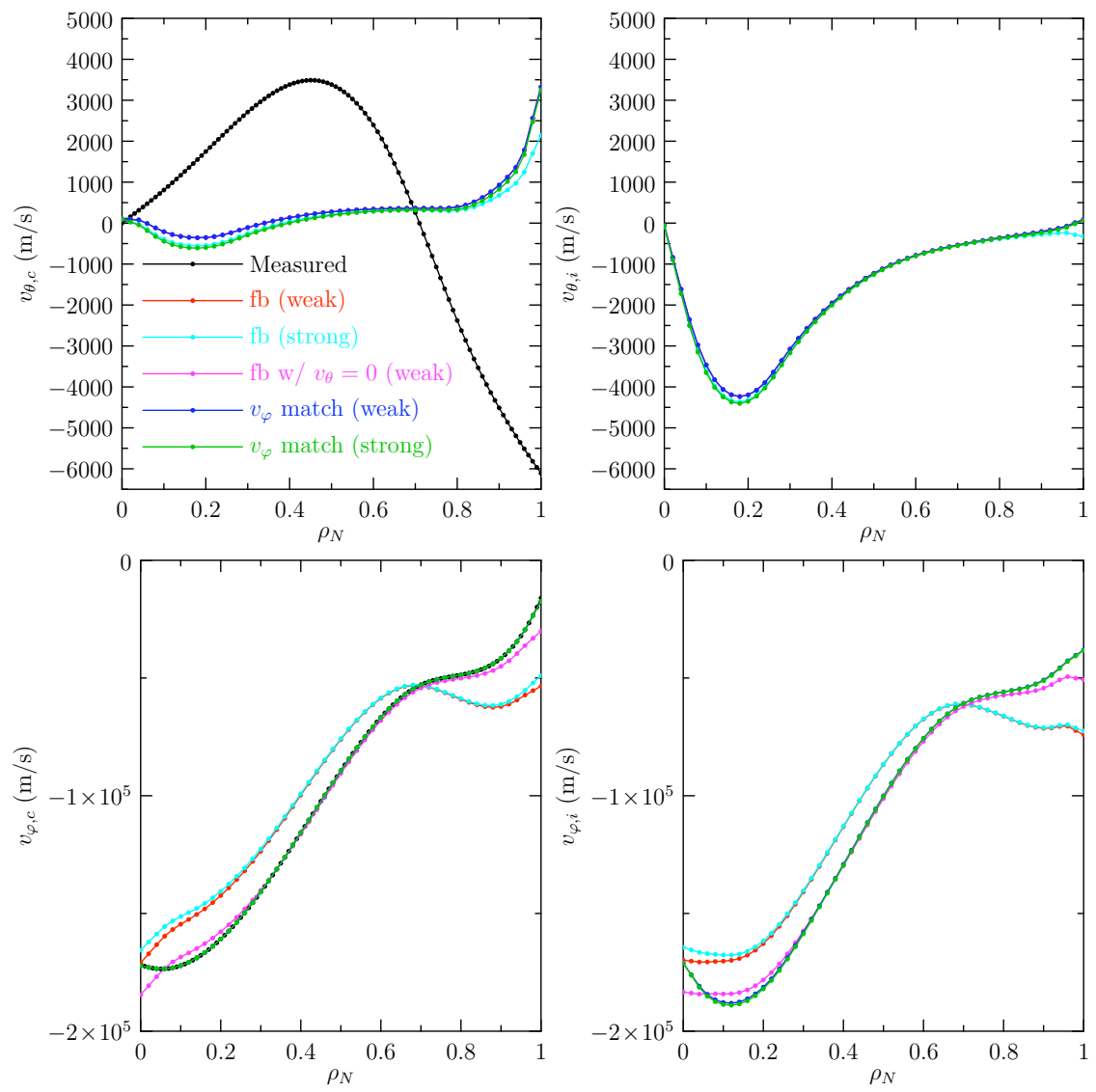

Figure 2: The corresponding NEO-computed carbon and deutrium toroidal and poloidal velocity profiles for DIII-D L-mode shot \#101391 using the VGEN-computed $E_{r}$. The measured flows are available only for carbon. In the weak rotation limit, the poloidal flows do not depend on $E_{r}$. For VGEN method " $v_{\varphi}$ match", the NEO-computed carbon $v_{\varphi}$ is forced to match the measured value. 


\title{
ESL/GA Progress Report
}

\author{
E. A. Belli, J. Candy, P. B. Snyder
}

March 2010

Further analysis of the DIII-D HEP benchmarking case has been done with NEO, focusing on the local physics. Comparisons with analytic theory for this more realistic plasma are consistent with our previous comparisons for a simple $s$ - $\alpha$ test case. Specifically, as shown in figure 1, we still find that Chang-Hinton theory overestimates the ion diffusivity over the entire profile. The deviation in the pedestal region is about $35 \%$. The Sauter model actually follows the NEO results quite well, only slightly underestimating the bootstrap current, with the largest deviation ( $30 \%$ ) seen near the magnetic axis. Surprisingly, the Sauter model shows only a $4 \%$ error in the pedestal region, though we believe that this is fortuitous. Hinton-Hazeltine theory generally underestimates both $\chi_{i}$ and $j_{\|}$, though the qualitative trends are correctly captured. Small differences were found in the NEO local transport results in the near-edge region $\left(\rho_{N}>0.8\right)$ with geometry model, collision model, and electron model. Specifically, using the general equilibrium with up-down asymmetry provides a $2-4 \%$ correction compared to the Miller equilibrium results. Using the zeroth-order Hirshman-Sigmar model provides a $17 \%$ correction compared with using the less accurate Connor model. Note that this conclusion is based on simulations which include the carbon impurities and it had previously been found that the deceleration effect due to dynamic friction, which is included in the zeroth-order Hirshman-Sigmar but not in the Connor model, is important for species of similar masses. Also, we found that including the complete kinetic electron physics provides a $5-10 \%$ correction compared to the adiabatic electron case.

Collisions have been added to the new GYRO field eigensolver. For simplicity, we have for now included only the pitch-angle scattering operator, as we have previously verified that the corrections on the gyrokinetic stability 
and transport due to the momentum-conserving term, which is complicated to implement due to the energy coupling it introduces, are negligible (see our May 2009 report). Nevertheless, even with just the Lorentz operator, the inclusion of collisions significantly increases the computational expense, since the inversion of the matrix representing the LHS of the gyrokinetic equation is now coupled in velocity space. For both the collisional case and the collisionless case, the solver has been optimized, using BLAS and LAPACK for all expensive matrix operations (LU decomposition, inverse, matrix-matrix multiply), and it is parallelized. Studies of our high-beta NSTX discharge case (\#132641) find that the collisions are stabilizing on the low $k_{\theta}$ hybrid ITG/KBM mode, and, even with large collision frequency, no dominant or sub-dominant micro-tearing modes are observed for this case. 

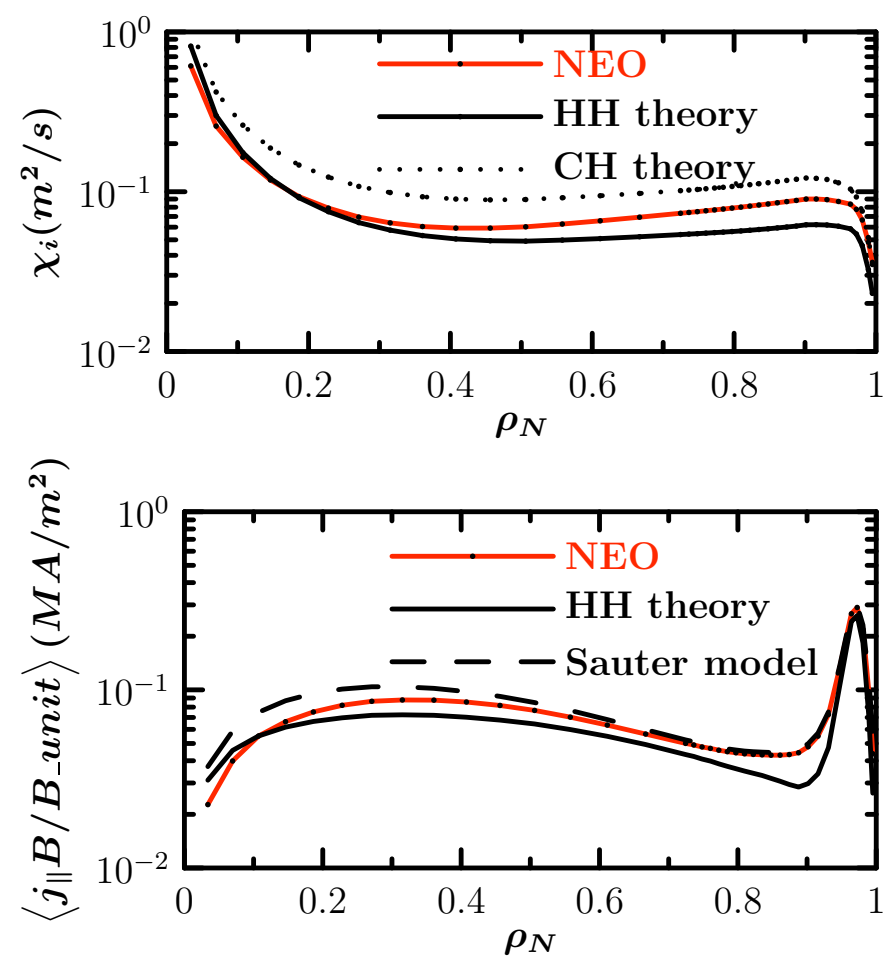

Figure 1: The second-order deuterium thermal diffusivity and the first-order bootstrap current vs. the normalized poloidal flux for DIII-D H-mode discharge \#98889, comparing the NEO results with Hinton-Hazeltine theory, Chang-Hinton theory, and the Sauter model. The simulations do not include impurities, use general geometry, and use the zeroth-order Hirshman-Sigmar model. 


\title{
ESL/GA Progress Report
}

\author{
E. A. Belli, J. Candy, P. B. Snyder
}

June 2010

The NEO-based tool VGEN for computing $E_{r}$ and the poloidal and toroidal flows has been integrated into the GYRO/TGYRO/NEO experimental profiles tool iterdb2gyro. This will allow for the rotation input parameters from NEO to be used in GYRO and TGYRO and for the flows to be used as a diagnostic in TGYRO. The PEQDSK format for the density and temperature profile data, which is used as input for ELITE, has also been added to iterdb2gyro. The PEQDSK file and GEQDSK geometry file form a complete set for generating the GYRO/TGYRO/NEO INPUT_profiles for a pure plasma. Extensions of the PEQDSK to include impurity profile data and the measured velocity flows are being considered. Re-analysis of finite-orbit-width effects on the neoclassical transport in the DIII-D Hmode pedestal using these more accurate profiles and including the general geometry effects is in progress.

Further analysis of the DIII-D L-mode deuterium parallel flows in the edge has focused on distinguishing the neoclassical and classical (diamagnetic) components and on an apparent correlation between the trend of the deuterium ion and carbon impurity flows at $\theta=255^{\circ}$ (near the bottom of the tokamak) but not at $\theta=0^{\circ}$ (outboard midplane). Here we define the components of $u_{\|}$as

$$
\begin{aligned}
& u_{E}=-\frac{c I}{B} \frac{d \Phi}{d \psi} \\
& u_{p}=-\frac{c I}{B} \frac{1}{z_{a} e n_{0 a}} \frac{d p_{0 a}}{d \psi} \\
& u_{k}=\frac{K_{a}}{n_{0 a}} B,
\end{aligned}
$$


such that $u_{\|}=u_{E}+u_{p}+u_{k}$. The results are shown in figures 1 and 2 for low and high density cases respectively. Recall that it was previously found that the trend of the NEO data for these cases qualitatively approaches the deuterium ion measurements outside the separatrix as $\rho_{N} \rightarrow 1$. For the deuterium ions, the neoclassical component, $u_{k}$, is small. For the low density shot, the edge ion $u_{\|}$is mostly determined by the pressure gradient component, $u_{p}$, while for the high-density shots the pressure gradient and $E_{r}$ components are competing. This is true at both poloidal locations. Moving from $\theta=0^{\circ}$ to $\theta=255^{\circ}, B$ (which can clearly be seen in the above equations as determining the poloidal dependence of $u_{\|}$) increases so the magnitudes of $u_{E}$ and $u_{p}$ become smaller and the magnitude of $u_{k}$ becomes larger. It appears that this is what is causing the less steep rise in $u_{\|}$in the edge for $\theta=255^{\circ}$, i.e. the $-u_{p}$ part, which is positive, is becoming smaller and the $-u_{E}$ part, which is negative, is becoming larger in absolute terms. The neoclassical component $u_{k}$ is negligible, specifically almost 100 times smaller than $u_{p}$ for the high-density case and more like 10 times smaller for the low-density case. For the latter, $-u_{k}$ is negative in the edge, so the $B$ factor is bringing down the $u_{\|}$even more. It is not yet clear whether there is any physics correlation between the ions and carbon since, while the carbon $u_{\|}$is mostly determined by the $E_{r}$ component in the core, in the edge all three parts are largely contributing. For carbon, even if the $n$ and $T$ profiles were the same as for the ions (which they are not), the $u_{p}$ influence is reduced by the $1 / Z$ factor, which allows the other components to be more competitive. The $u_{E}$ component is the same as for the ions, but the neoclassical part is much larger. Theoretically, it is also known that the primary driving dynamics for the two species are quite different, i.e. in the very heavy ion impurity limit, the impurity dynamics are mostly determined by self-collisions while the light ion dynamics are mostly determined by the cross-species collision coupling. Overall, similar results were found in the co-NBI phase (the data in figures 1 and 2 is during the counter-NBI phase), as well as for ECH-injected plasmas, and the main conclusion is that the carbon velocities do not fully reflect the deuterium velocities in the edge. 

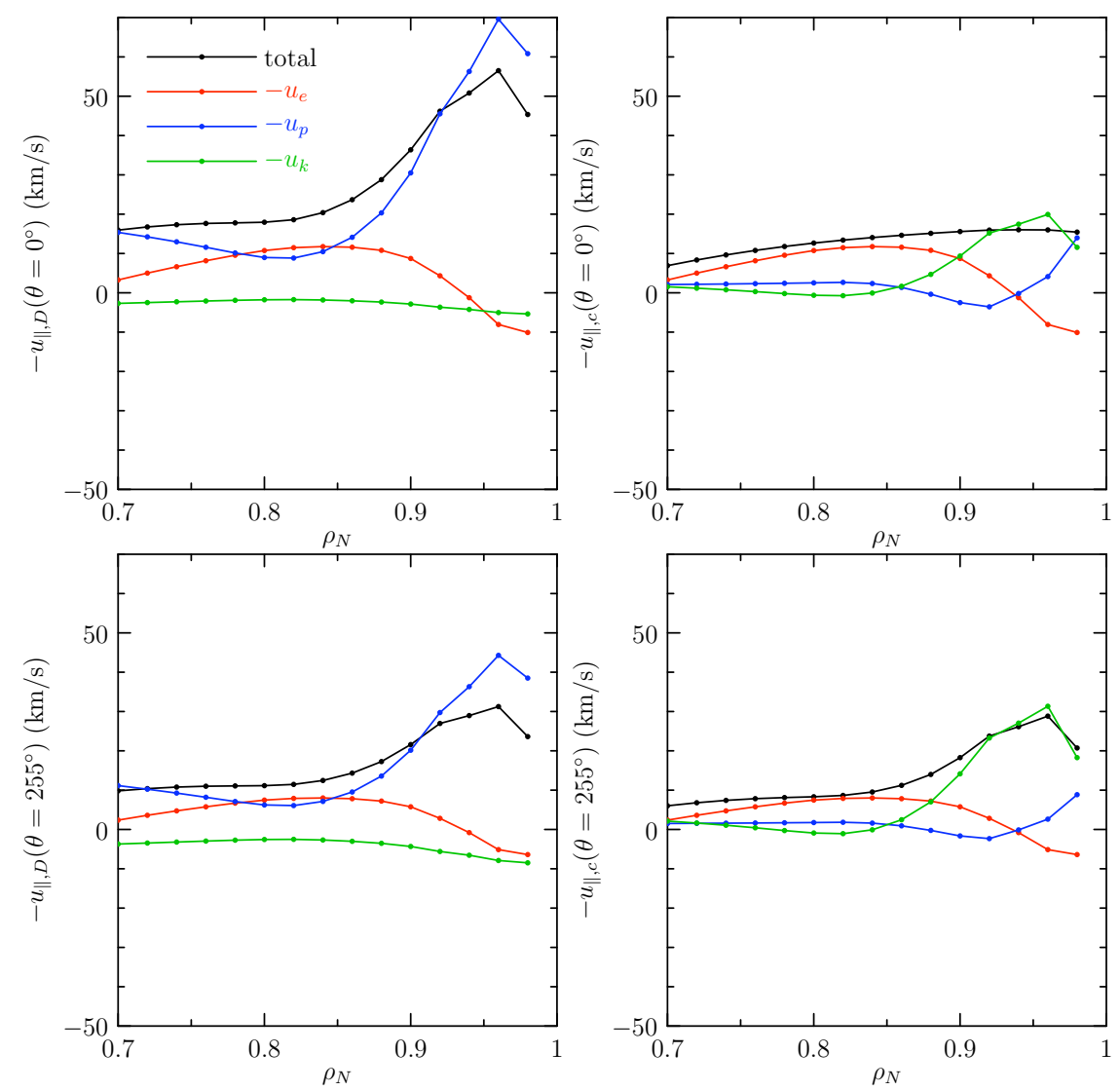

Figure 1: Radial profiles of the deuterium ion and carbon impurity parallel flows from NEO for low-density DIII-D L-mode shot \#134046 at $\mathrm{t}=2.5 \mathrm{~s}$ $\left(n_{e}=1.8 e 13 \mathrm{~cm}^{-3}\right)$ at $\theta=0^{\circ}$ (outboard midplane) and $\theta=255^{\circ}$ (near the bottom of the tokamak). The radial electric field $\left(u_{e}\right)$, pressure gradient $\left(u_{p}\right)$, and neoclassical components $\left(u_{k}\right)$ are also shown. 

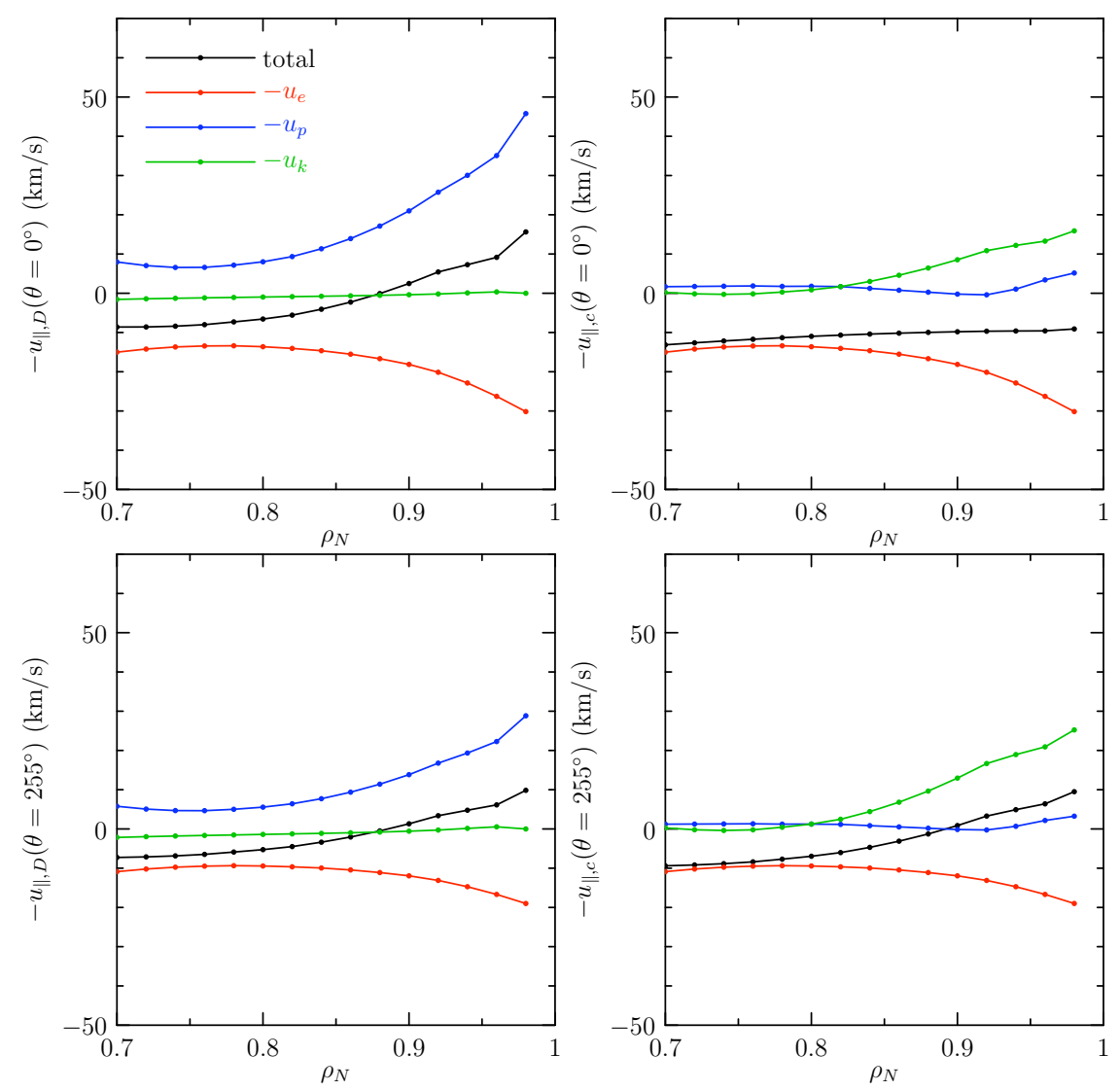

Figure 2: Radial profiles of the deuterium ion and carbon impurity parallel flows from NEO for high-density DIII-D L-mode shot \#134046 at $\mathrm{t}=2.5 \mathrm{~s}$ $\left(n_{e}=3 e 13 \mathrm{~cm}^{-3}\right)$ at $\theta=0^{\circ}$ (outboard midplane) and $\theta=255^{\circ}$ (near the bottom of the tokamak). The radial electric field $\left(u_{e}\right)$, pressure gradient $\left(u_{p}\right)$, and neoclassical components $\left(u_{k}\right)$ are also shown. 


\title{
ESL/GA Progress Report
}

\author{
E. A. Belli, J. Candy, P. B. Snyder
}

July 2010

The effects of orbit squeezing on the neoclassical transport in the core and edge are being studied with NEO via solution of the hierarchy of Hazeltine drift-kinetic equations ordered in $\rho_{* i}$. For verification and insight, an analytic solution is being derived for the simple case of a single ion species and zero temperature gradient. The first-order solution is given by

$$
f_{1}=F \frac{v_{\|}}{\Omega_{c}},
$$

where

$$
F(r, \varepsilon)=-\frac{I}{\psi^{\prime}} f_{0}\left(\frac{d \ln n_{0}}{d r}+\frac{z e}{T_{0}} \frac{d \Phi_{0}}{d r}\right) .
$$

For the second-order solution, we assume $s$ - $\alpha$ geometry for simplicity. While the first-order solution is independent of the collisional regime, the higherorder solutions are not. In the banana regime, we find the second-order solution is given as follows to lowest-order in $\epsilon$ and $\nu$ :

$$
f_{2} \sim G\left[-\frac{1}{2} \frac{v_{\|}^{2}}{\Omega_{c}^{2}}-\frac{\mu}{B_{0}} \Omega_{c}^{2}+\frac{\varepsilon}{\Omega_{c 0}^{2}}\left(1+\frac{5}{6} \epsilon^{2}\right)\right] .
$$

In the Pfirsch-Schlüter regime, we find the second-order solution is given as follows to lowest-order in $1 / \nu$ :

$$
f_{2} \sim-G \frac{\varepsilon}{\Omega_{c 0}^{2}} \frac{4}{5}\left(\frac{B_{0}^{2}}{B^{2}}-1-\frac{3}{2} \varepsilon^{2}\right) .
$$

Here we have defined

$$
G(r, \varepsilon)=\frac{I}{\psi^{\prime}}\left(\frac{\partial F}{\partial r}+\frac{z e}{T_{0}} \frac{d \Phi_{0}}{d r} F\right) .
$$


Figures 1 and 2 show a comparison of the NEO results and the analytic formulas. Good agreement is found in both collision limits.

The transport coefficients from the second-order solution are zero for up-down symmetric plasmas and thus the third-order solution is needed to study higher-order finite-orbit-width and orbit squeezing effects. However, the third-order solution is not yet well-understood in an analogous way due to the difficulty with analytic treatment of the collisional dynamics. Nevertheless, the approximations needed to isolate the orbit squeezing factor $\left(\propto \frac{\partial^{2} \Phi_{0}}{\partial r^{2}} \frac{a}{L_{n}}\right)$ are being explored and the dominate higher-order effects in DIII-D L-mode and H-mode plasmas will be studied. 

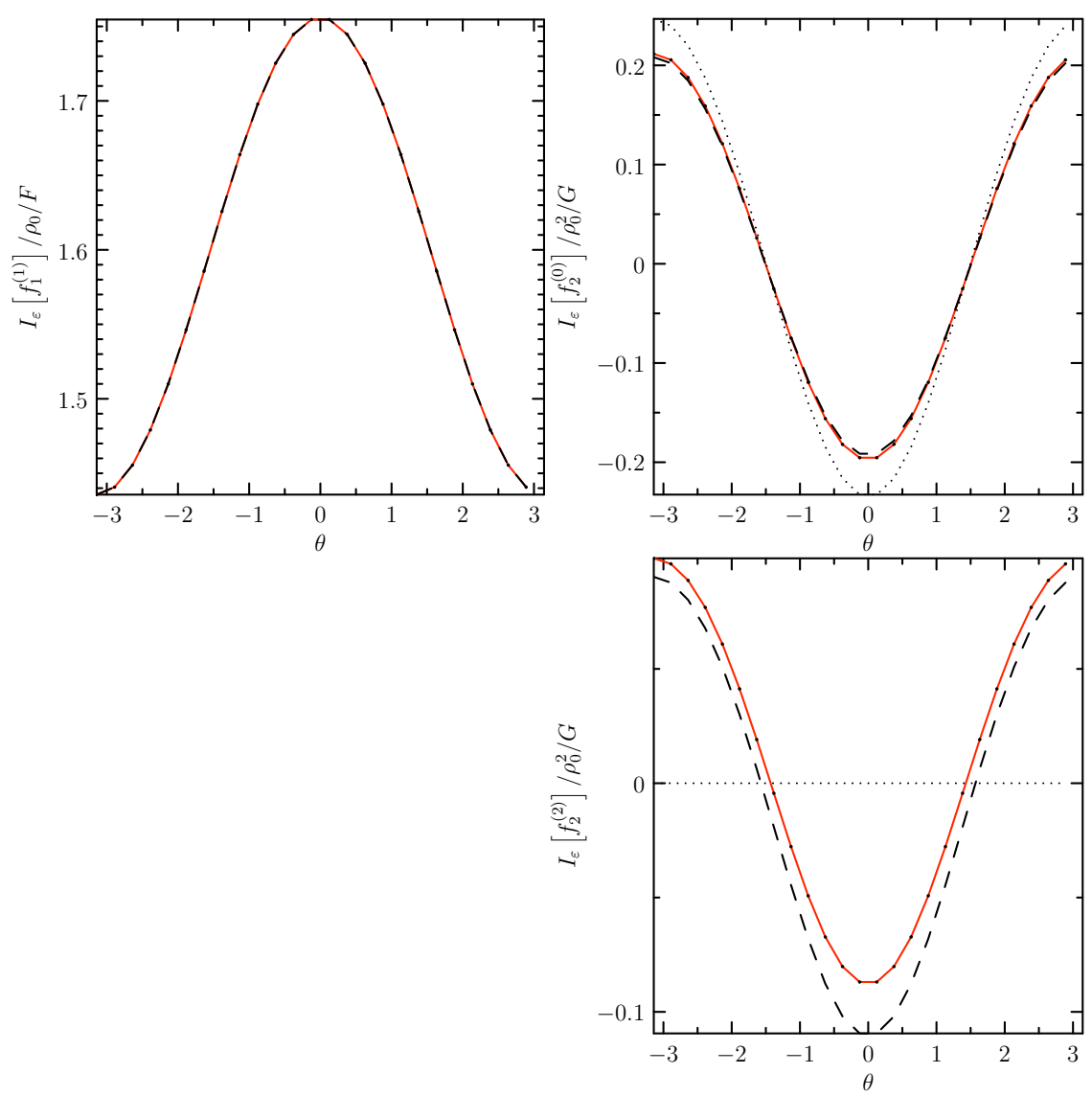

Figure 1: Density moment of the $P_{1}$ component of the first-order distribution function (left) with the analytic solution (black dashed line) and the $P_{0}$ and $P_{2}$ components of the second-order distribution function (right top and right bottom) with the analytic approximations in the banana regime (black dashed line) and in the Pfirsch-Schlüter regime (black dotted line). Here the $P_{n}$ 's are Legendre polynomials in $\xi$. The NEO results are given by the red curves. The parameters for this case are: $s$ - $\alpha$ geometry, $R_{0} / a=5$, $\rho_{0 i}=0.001, q(r)=3 r / a$ (such that $I / \psi^{\prime}$ is constant), $\frac{\partial \Phi_{0}}{\partial r}(r)=20(r / a-0.5)$, $n_{0}(r)=\exp (-r / a), T_{0}(r)=1$. A single ion species is simulated and the full Hirshman-Sigmar collision operator is used. These results are for the banana regime, $\left(a / v_{t i}\right) \tau_{i i}^{-1}=1 \mathrm{e}-4$. 

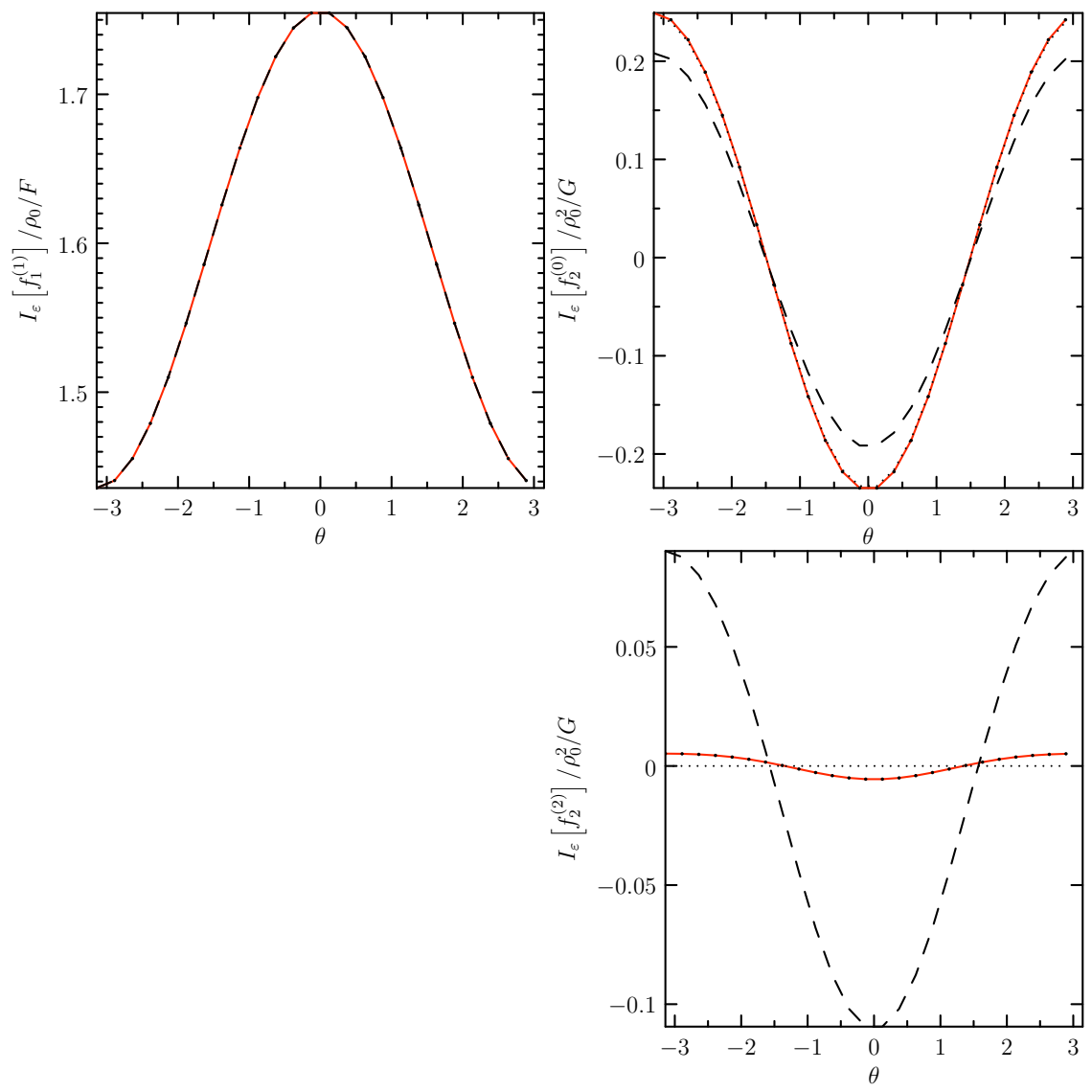

Figure 2: Density moment of the $P_{1}$ component of the first-order distribution function (left) with the analytic solution (black dashed line) and the $P_{0}$ and $P_{2}$ components of the second-order distribution function (right top and right bottom) with the analytic approximations in the banana regime (black dashed line) and in the Pfirsch-Schlüter regime (black dotted line). The NEO results are given by the red curves. The parameters are the same as for figure 1 but in the highly collisional regime, $\left(a / v_{t i}\right) \tau_{i i}^{-1}=1$. 


\title{
ESL/GA Progress Report
}

\author{
E. A. Belli, J. Candy, P. B. Snyder
}

August 2010

An analytic solution for the hierarchy of distribution functions is being derived to verify the NEO implementation of higher-order finite-orbit-width terms and to develop insight regarding the scaling of the neoclassical transport due to orbit squeezing. We consider a simple case of a single ion species with uniform temperature (i.e. zero temperature gradient) and $s-\alpha$ geometry. For the collision operator, we consider HS0. The first-order and second-order distribution functions for these cases were given in the July report for both the Pfirsch-Schlüter and banana collisional regimes. However, for the third-order solution we focus only on the former, since we found a simple analytic solution in the banana regime to be intractable.

We consider the hierarchy of drift-kinetic equations in $(\varepsilon, \xi)$ velocityspace coordinates and, using an expansion of the distribution function in Legendre polynomials $P$ in $\xi$, we terminate the series of equations at $P_{2}$, due to the large collision frequency limit. First, to test the method, we have derived an analytic formula for the first-order distribution function in this regime for finite temperature gradient. The solution for the $P_{0}, P_{1}$, and $P_{2}$, components is given as follows to lowest order in $\epsilon$ and $1 / \tau_{i i}^{-1}$ :

$$
\begin{aligned}
g_{1}^{(0)} & =-\frac{\mathcal{J}_{\psi} B}{\sqrt{2 \varepsilon} v_{t a}} \epsilon \sin (\theta)\left[S_{t} \sqrt{2} C\left(\varepsilon^{3 / 2}\right)+C\left(c_{1}(\varepsilon)\right)\right] \\
g_{1}^{(1)} & =-\sqrt{2} \frac{B_{0}}{B}\left(S_{n} \varepsilon^{1 / 2}+S_{t} \varepsilon^{3 / 2}\right)+c_{1}(\varepsilon) \frac{B}{B_{0}} \\
g_{1}^{(2)} & =-\frac{\sqrt{2 \varepsilon} v_{t a}}{3 \nu_{d} \mathcal{J}_{\psi} B} \frac{1}{B_{0}} \frac{\partial B}{\partial \theta} c_{1}(\varepsilon)
\end{aligned}
$$


where the source terms are given by

$$
\begin{aligned}
S_{n} & =\frac{I}{\psi^{\prime}} \frac{v_{t a}}{\Omega_{c a 0}}\left(\frac{\partial \ln n}{\partial r}+\frac{z_{a} e}{T_{0 a}} \frac{\partial \Phi_{0}}{\partial r}-\frac{3}{2} \frac{\partial \ln T}{\partial r}\right) \\
S_{t} & =\frac{I}{\psi^{\prime}} \frac{v_{t a}}{\Omega_{c a 0}} \frac{\partial \ln T}{\partial r}
\end{aligned}
$$

and the constant is found to be

$$
c_{1}(\varepsilon)=S_{t} \sqrt{2}\left[\varepsilon^{3 / 2}-\varepsilon^{1 / 2} \frac{I_{\varepsilon}\left(\varepsilon^{3} / \nu_{d}\right)}{I_{\varepsilon}\left(\varepsilon^{2} / \nu_{d}\right)}\right] .
$$

In the limit of zero temperature gradient, only the $P_{1}$ component is nonzero and we recover our previous result. With finite temperature gradient, we find that the ion parallel flow coefficient is $k_{i}=-2.37$. Note that this differs from the reported value of -1.6 for the full linearized operator. A comparison of NEO with the analytic results is shown in figures 1 and 2 . Here NEO uses 18 Legendre polynomials. The agreement becomes better if only 3 Legendre polynomials are used in the numerical calculation, though it is quite good also with the full spectrum.

An analogous result has been derived for the third-order distribution function in the zero temperature gradient limit. Comparisons with NEO are still in progress. 

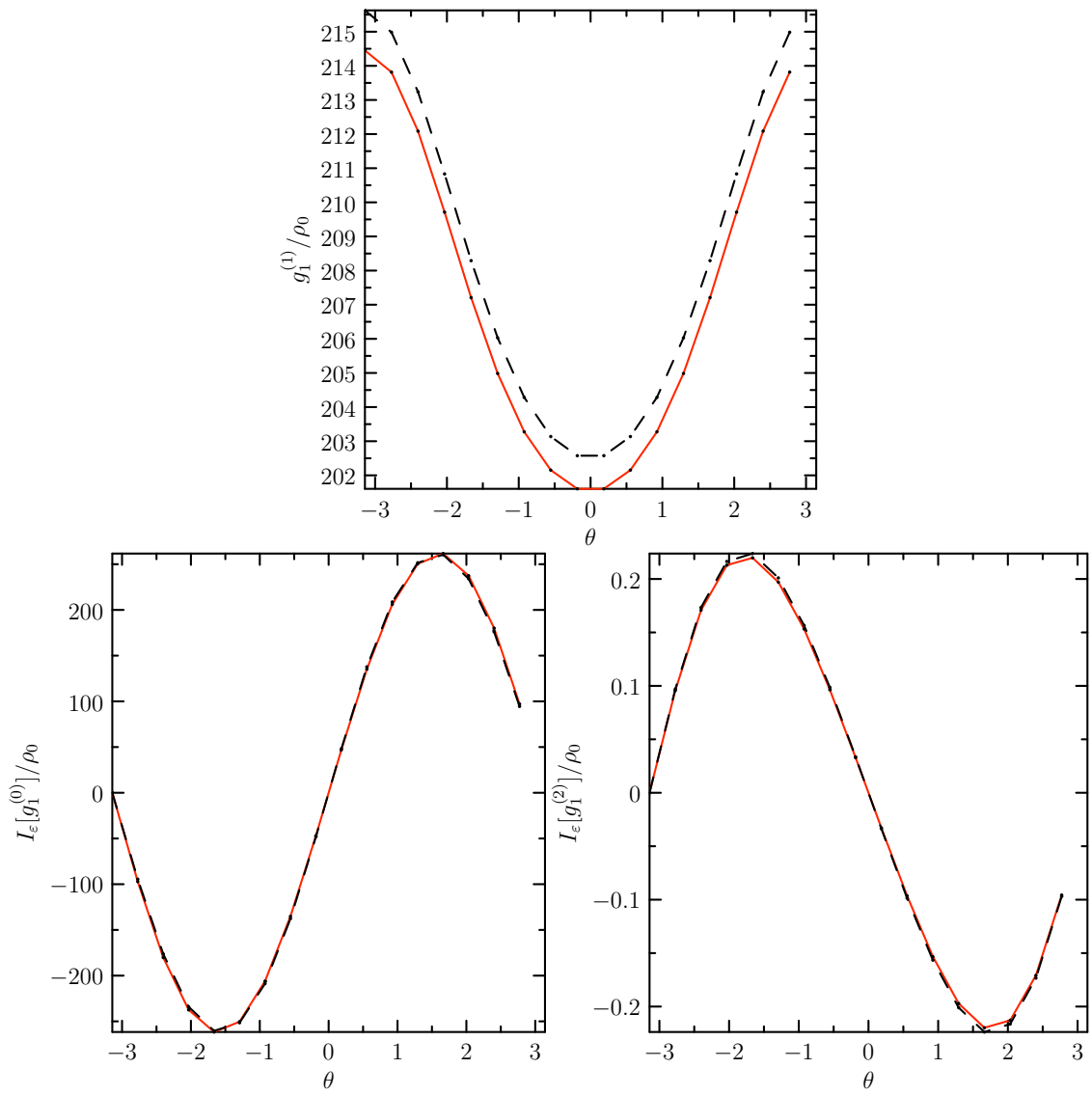

Figure 1: Density moment of the $P_{1}, P_{0}$, and $P_{2}$ components of the first-order distribution function comparing the NEO results (red) with the analytic theory in the the Pfirsch-Schlüter regime (black dashed lines) at $r / a=$ 0.5. The parameters for this case are: $s-\alpha$ geometry, $R_{0} / a=5, \rho_{0 i}=0.001$, $q(r)=3 r / a$ (such that $I / \psi^{\prime}$ is constant), $\frac{\partial \Phi_{0}}{\partial r}(r)=0, a / L_{n}=2, a / L_{T}=2$. A single ion species is simulated and the zeroth-order Hirshman-Sigmar collision operator is used. These results are for the highly-collisional regime, $\left(a / v_{t i}\right) \tau_{i i}^{-1}=10$. 

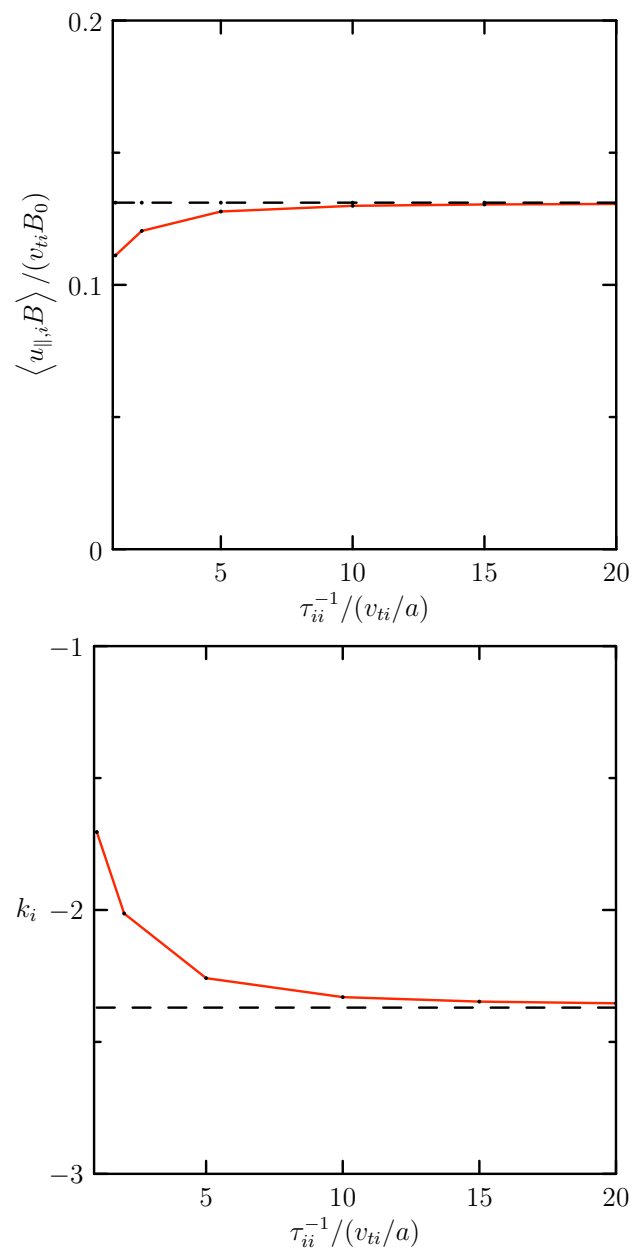

Figure 2: The ion parallel flow and ion parallel flow coefficient vs. collision rate comparing the NEO results (red) with the analytic theory in the the Pfirsch-Schlüter regime (black dashed lines) at $r / a=0.5$. 


\title{
ESL/GA Progress Report
}

\author{
E. A. Belli, J. Candy, P. B. Snyder
}

September 2010

An analytic solution for the third-order distribution function describing non-local neoclassical transport in the Pfirsch-Schlüter regime has been derived. We consider the hierarchy of drift-kinetic equations in $(\varepsilon, \xi)$ coordinates and use an expansion of the distribution function in Legendre polynomials in $\xi$, terminating the series of equations at $P_{2}$ due to the large collision frequency limit. For simplicity, we assume a single ion species, $s$ - $\alpha$ geometry, zero temperature gradient, and the zeroth-order HirshmanSigmar collision model. Using the second-order solution given in our July 2010 report, we find that the solution for the $P_{0}, P_{1}$, and $P_{2}$ components of the third-order distribution function is as follows to first order in $1 / \tau_{i i}^{-1}$ and to second-order in $\epsilon$ :

$$
\begin{aligned}
f_{3}^{(0)}= & \frac{1}{\Omega_{0}^{3}} \frac{\mathcal{J}_{\psi} B}{\sqrt{\varepsilon}} \frac{2}{5} C_{1}\left(\varepsilon^{3 / 2}\right)\left[H \epsilon^{2} \sin (2 \theta)-6 \frac{I}{\psi^{\prime}} \frac{\epsilon^{2}}{R_{0}} G \sin (\theta)\right] \\
f_{3}^{(1)}= & \frac{1}{\Omega_{0}^{3}} \frac{4}{5 \sqrt{2}}\left\{H\left[\frac{B_{0}^{3}}{B^{3}}-2 \frac{B}{B_{0}}\left(1+\frac{3}{2} \epsilon^{2}\right)\right]-H K \frac{5}{2} \epsilon^{2} \frac{B}{B_{0}} \varepsilon^{1 / 2}\right. \\
& +6 \frac{I}{\psi^{\prime}} \frac{\epsilon}{R_{0}} G\left[-\frac{B_{0}}{B} \varepsilon^{3 / 2}+\frac{B}{B_{0}}\left(1-\frac{1}{2} \epsilon^{2}\right)\left(\varepsilon^{3 / 2}-K \varepsilon^{1 / 2}\right)\right] \\
& \left.+\frac{I}{\psi^{\prime}} \frac{z e}{m} \frac{\partial \Phi_{0}}{\partial r} G \varepsilon^{1 / 2}\left[\frac{1}{2} \frac{B_{0}^{3}}{B^{3}}+2 \frac{B_{0}}{B}\left(1+\frac{3}{2} \epsilon^{2}\right)+\frac{3}{2} \frac{B}{B_{0}}\right]\right\} \\
f_{3}^{(2)}= & \frac{1}{\Omega_{0}^{3}}\left(\frac{4}{15} \frac{1}{\nu_{D} \mathcal{J}_{\psi} B}\right) \frac{1}{B_{0}} \frac{\partial B}{\partial \theta}\left\{H\left[\left(\frac{B_{0}^{4}}{B^{4}}-1-\epsilon^{2}\right) \varepsilon^{2}+\frac{5}{2} K \epsilon^{2} \varepsilon\right]\right. \\
& \left.-6 \frac{I}{\psi^{\prime}} \frac{\epsilon}{R_{0}} G\left(\varepsilon^{2}-K \varepsilon\right)+\frac{3}{2} \frac{z}{\psi^{\prime}} \frac{z e}{m} \frac{\partial \Phi_{0}}{\partial r} G\left(\frac{B_{0}^{4}}{B^{4}}-1\right) \varepsilon\right\},
\end{aligned}
$$


where $K v_{t}^{2}=\left(\int_{0}^{\infty} d \varepsilon e^{-\varepsilon / v_{t a}^{2}} \varepsilon^{7 / 2} \nu_{D}^{-1}\right) /\left(\int_{0}^{\infty} d \varepsilon e^{-\varepsilon / v_{t a}^{2}} \varepsilon^{5 / 2} \nu_{D}^{-1}\right)$ and the equilibriumscale source functions are given by

$$
\begin{aligned}
F(r, \varepsilon) & =-\frac{I}{\psi^{\prime}} f_{0}\left(\frac{d \ln n_{0}}{d r}+\frac{z e}{T_{0}} \frac{d \Phi_{0}}{d r}\right) \\
G(r, \varepsilon) & =\frac{I}{\psi^{\prime}}\left(\frac{\partial F}{\partial r}+\frac{z e}{T_{0}} \frac{d \Phi_{0}}{d r} F\right) \\
H(r, \varepsilon) & =\frac{I}{\psi^{\prime}}\left(\frac{\partial G}{\partial r}+\frac{z e}{T_{0}} \frac{d \Phi_{0}}{d r} G\right) .
\end{aligned}
$$

For the $P_{0}$ component, the collision term is defined with respect to the zeroth-order Hirshman-Sigmar operator as

$$
C_{1}\left(\varepsilon^{3 / 2}\right)=-\nu_{s} \varepsilon^{3 / 2}+\nu_{s} \varepsilon^{1 / 2} \frac{\int_{0}^{\infty} d \varepsilon e^{-\varepsilon / v_{t}^{2}} \varepsilon^{5 / 2} \nu_{S}}{\int_{0}^{\infty} d \varepsilon e^{-\varepsilon / v_{t}^{2}} \varepsilon^{3 / 2} \nu_{S}}
$$

though the solution is also valid for the Connor model with $\nu_{S}=\nu_{D}$. Good agreement is found between the analytic theory and the NEO results, as shown in figure 1. From the analytic solution, we can compute the thirdorder parallel flow:

$$
\left\langle\frac{u_{\|}^{3}}{v_{t a}} \frac{B}{B_{0}}\right\rangle \frac{1}{\rho_{0 a}^{3}}=-\frac{H}{f_{0}} K \epsilon^{2}-\frac{G}{f_{0}} \frac{12}{5} \frac{I}{\psi^{\prime}} \frac{\epsilon}{R_{0}} K+\frac{I}{\psi^{\prime}} \frac{z e}{T_{0}} \frac{\partial \Phi_{0}}{\partial r} \frac{G}{f_{0}}\left(\frac{8}{5}+\frac{9}{5} \epsilon^{2}\right) .
$$

Noting that the usual "orbit-squeezing" factor $\left(\propto \frac{\partial^{2} \Phi_{0}}{\partial r^{2}} \frac{a}{L_{n}}\right)$ is contained within $H$, we thus find that the flow is $\mathcal{O}\left(\epsilon^{2}\right)$ smaller in this factor than the Hinton-Kim banana regime theory.

Both our banana regime and Pfirsch-Schlüter regime higher-order analytic calculations have neglected the nonlinear collision operator without justification. This is also done in the NEO implementation of the hierarchy of equations. However, re-analysis shows that inclusion of the nonlinear collision is crucial. For example, for the second-order solution in the banana regime, we find that

$$
f_{2}=\frac{1}{\Omega_{0}^{2}}\left(\frac{2}{3} \varepsilon-\mu B_{0}\right) \frac{F^{2}}{f_{0}}+\frac{1}{\Omega_{0}^{2}}\left(-2 \varepsilon+\mu B_{0}\right) \epsilon \cos (\theta) G
$$

Thus, comparing this with our previous solution, we find that it is $\mathcal{O}(\epsilon)$ larger. The solubility condition essentially removes the $\theta$-average of the collisionless part of the solution, thus reducing its contribution to $\mathcal{O}(\epsilon)$. 
The largest component then results from the nonlinear collision dynamics. Similarly, in the Pfirsch-Schlüter regime, we find that the solution is

$$
f_{2}=-\frac{\varepsilon}{\Omega_{0}^{2}}\left(\frac{B_{0}^{2}}{B^{2}}-1-\frac{3}{2} \epsilon^{2}\right)\left(\frac{4}{5} G+\frac{7}{15} \frac{F^{2}}{f_{0}}\right)+\frac{\varepsilon}{\Omega_{0}^{2}} \frac{2}{3} \frac{B_{0}^{2}}{B^{2}} \frac{F^{2}}{f_{0}} P_{2}(\xi) .
$$

An upgrade of NEO to implement the full linearized Fokker-Planck collision operator and to implement explicit evaluation of the nonlinear operator in the higher-order equations is in progress. 

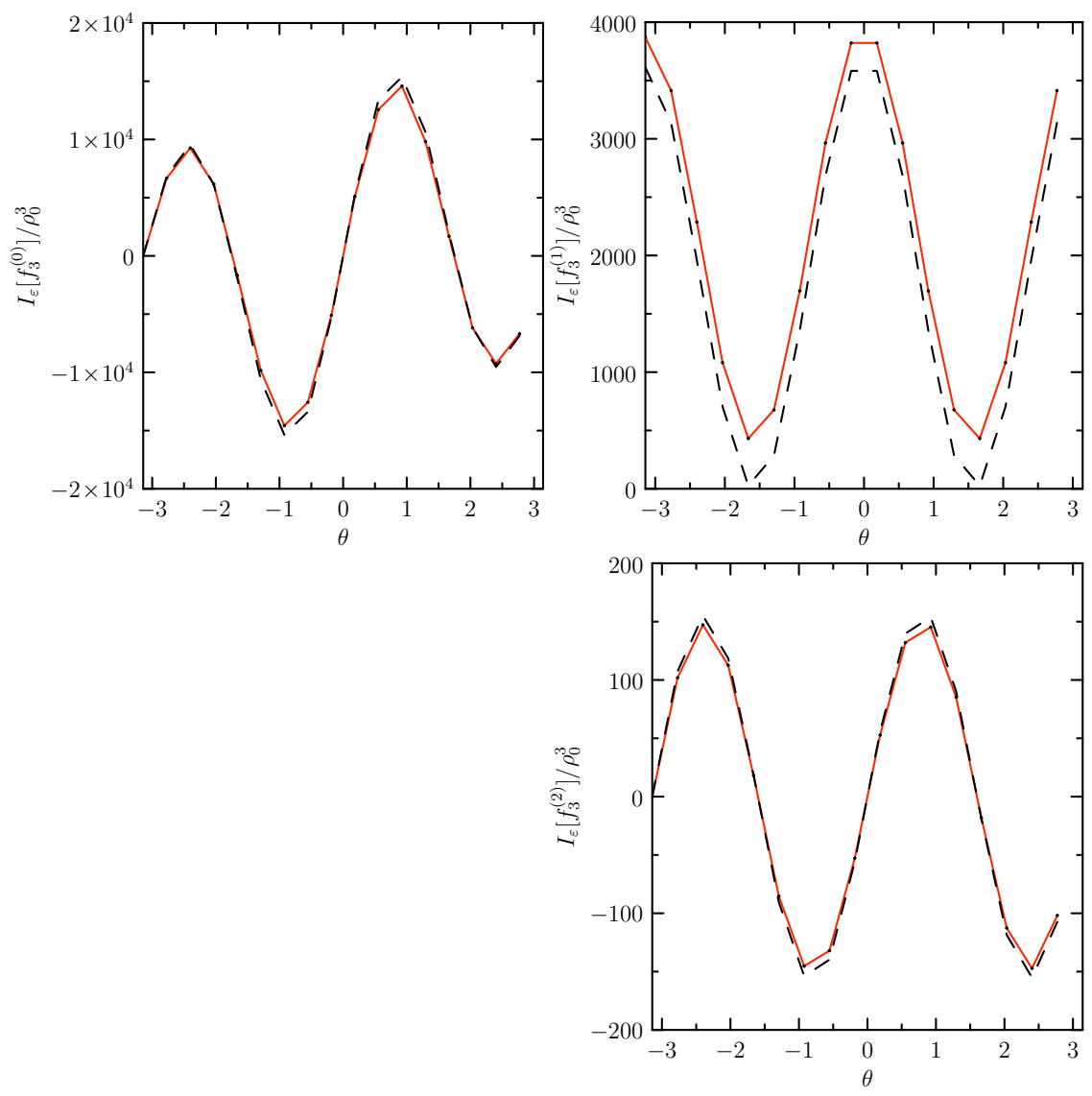

Figure 1: Density moment of the $P_{1}, P_{0}$, and $P_{2}$ components of the thirdorder distribution function comparing the NEO results (red) with the analytic theory in the the Pfirsch-Schlüter regime (black dashed lines) at $r / a=0.5$. The parameters for this case are: $s$ - $\alpha$ geometry, $R_{0} / a=5$, $\rho_{0 i}=0.001, q(r)=3 r / a$ (such that $I / \psi^{\prime}$ is constant), $\frac{\partial \Phi_{0}}{\partial r}(r)=2(r / a-0.5)$, $n_{0}(r)=2 e^{-r / a}, T_{0}(r)=1,\left(a / v_{t i}\right) \tau_{i i}^{-1}=10$. A single ion species is simulated and the zeroth-order Hirshman-Sigmar collision operator is used. 


\title{
ESL/GA Progress Report
}

\author{
E. A. Belli, J. Candy, P. B. Snyder
}

October 2010

NEO is being upgraded to implement the full linearized Fokker-Planck collision operator. We retain an expansion of Legendre polynomials in $\xi$, but for energy, we assume a monomial expansion in normalized velocity, $x_{a}=v /\left(\sqrt{2} v_{t a}\right)$. Thus, we expand the $\mathrm{n}$-th order perturbed distribution function as

$$
f_{n a}=f_{0 a} \sum_{l=0}^{\infty} \sum_{m=0}^{\infty} \hat{f}_{n a}^{l m} P_{l}(\xi) x_{a}^{\alpha m+\beta l},
$$

where optimal values of the integers $\alpha$ and $\beta$ will be determined. With this expansion and applying the integral

$$
I \equiv\left(\frac{n_{0 a}}{(2 \pi)^{3 / 2} v_{t a}^{3}}\right)^{-1}\left(\frac{2 l+1}{2}\right) \int_{-1}^{1} d \xi P_{l}(\xi) \int_{0}^{\infty} d x_{a} x_{a}^{\alpha m+\beta l+2},
$$

the collision operator collocation integrals for the test and field particle operator components can be written as

$$
\begin{gathered}
I\left[C_{a b}^{T}\left(f_{1 a}, f_{0 b}\right)\right]=\tau_{a b}^{-1} \sqrt{\frac{d}{\pi}} \sum_{l^{\prime} m^{\prime}} \hat{f}_{1 a}^{l^{\prime} m^{\prime}} \delta_{l l^{\prime}}\{ \\
-l(l+1)\left[F\left(d, \alpha\left(m+m^{\prime}\right)+2 \beta l-1,0\right)-F\left(d, \alpha\left(m+m^{\prime}\right)+2 \beta l-3,2\right)\right] \\
+4(\alpha m+\beta l)\left[\left(1-\frac{T_{0 a}}{T_{0 b}}\right) F\left(d, \alpha\left(m+m^{\prime}\right)+2 \beta l-1,2\right)\right. \\
\left.\left.-\frac{1}{2}\left(\alpha m^{\prime}+\beta l\right) F\left(d, \alpha\left(m+m^{\prime}\right)+2 \beta l-3,2\right)\right]\right\} \\
I\left[C_{a b}^{F}\left(f_{0 a}, f_{1 b}\right)\right]=\tau_{a b}^{-1} \frac{2}{\sqrt{\pi}} d^{3 / 2} \sum_{l^{\prime} m^{\prime}} \hat{f}_{1 b}^{l^{\prime} m^{\prime}} \delta_{l l^{\prime}} d^{(1 / 2)\left(\alpha m^{\prime}+\beta l\right)}\{ \\
\frac{m_{a}}{m_{b}}(1+d)^{-(1 / 2)\left(\alpha\left(m+m^{\prime}\right)+2 \beta l+3\right)} \Gamma\left[\frac{1}{2}\left(\alpha\left(m+m^{\prime}\right)+2 \beta l+3\right)\right]
\end{gathered}
$$




$$
\begin{gathered}
-\frac{2}{l+1 / 2}\left[\frac{m_{a}}{m_{b}}-l\left(1-\frac{m_{a}}{m_{b}}\right)\right] F\left(d, \alpha m+\beta l-l+1, \alpha m^{\prime}+\beta l+l+2\right) \\
\begin{aligned}
-\frac{2}{l+1 / 2}[ & \left.1+l\left(1-\frac{m_{a}}{m_{b}}\right)\right] \bar{F}\left(d, \alpha m+\beta l+l+2, \alpha m^{\prime}+\beta l-l+1\right) \\
& -\frac{l(l-1)}{l^{2}-1 / 4} F\left(d, \alpha m+\beta l-l+3, \alpha m^{\prime}+\beta l+l+2\right) \\
& -\frac{l(l-1)}{l^{2}-1 / 4} \bar{F}\left(d, \alpha m+\beta l+l+2, \alpha m^{\prime}+\beta l-l+3\right) \\
+ & \frac{(l+1)(l+2)}{(l+3 / 2)(l+1 / 2)} F\left(d, \alpha m+\beta l-l+1, \alpha m^{\prime}+\beta l+l+4\right) \\
+ & \left.\frac{(l+1)(l+2)}{(l+3 / 2)(l+1 / 2)} \bar{F}\left(d, \alpha m+\beta l+l+4, \alpha m^{\prime}+\beta l-l+1\right)\right\}
\end{aligned}
\end{gathered}
$$

The first term in the test particle operator represents the Lorentz operator and the second term represents the energy diffusion. Note that both components are diagonal in $l$. Here we have defined $d=v_{t a}^{2} / v_{t b}^{2}$ and we have defined the double integral functions

$$
\begin{aligned}
& F(d, m, n)=\int_{0}^{\infty} d x e^{-x^{2}} x^{m} \int_{0}^{x} d y e^{-y^{2} d} y^{n}, \\
& \bar{F}(d, m, n)=\int_{0}^{\infty} d x e^{-x^{2}} x^{m} \int_{x}^{\infty} d y e^{-y^{2} d} y^{n} .
\end{aligned}
$$

Numerically stable algorithms for the calculation of the double integral functions, including for negative exponents, which can also maintain high accuracy in the limits of large and small $d$ (e.g. for electron-ion and ion-electron collisions) are being explored.

Similar expressions have been derived for the Connor operator and HS0 operator, which will also be implemented using the new energy expansion. For example, HSO contains the same Lorentz term and the following restoring terms:

$$
\begin{gathered}
I\left(R_{a b}\right)=\left(\frac{m_{b} n_{0 b} v_{t b}}{m_{a} n_{0 a} v_{t a}}\right) \tau_{a b}^{-1} \frac{T_{0 a}}{T_{0 b}}\left(1+\frac{m_{a}}{m_{b}}\right) \frac{4}{\sqrt{d \pi}} \frac{F(d, \alpha m+\beta, 2)}{F(d, 1,2)} \\
\sum_{l^{\prime} m^{\prime}} \hat{f}_{1 b}^{l^{\prime} m^{\prime}} \delta_{l, 1} \delta_{l^{\prime}, 1} F\left(1 / d, \alpha m^{\prime}+\beta, 2\right) \\
I\left(U_{a}\right)=\tau_{a b}^{-1} 2 \sqrt{\frac{d}{\pi}} \sum_{l^{\prime} m^{\prime}} \hat{f}_{1 a}^{l^{\prime} m^{\prime}} \delta_{l, 1} \delta_{l^{\prime}, 1}\left[F\left(d, \alpha\left(m+m^{\prime}\right)+2 \beta l-1,0\right)\right. \\
-F\left(d, \alpha\left(m+m^{\prime}\right)+2 \beta l-3,2\right) \\
\left.-2 \frac{T_{0 a}}{T_{0 b}}\left(1+\frac{m_{a}}{m_{b}}\right) F\left(d, \alpha\left(m+m^{\prime}\right)+2 \beta l-1,2\right)\right]
\end{gathered}
$$

The collisionless terms in the drift-kinetic equation are tri-diagonal in $l$. All of the energy collocation integrals for these terms and for the transport coefficients can be written in terms of $\Gamma$ functions. 


\title{
ESL/GA Progress Report
}

\author{
E. A. Belli, J. Candy, P. B. Snyder
}

November 2010

The NCLASS subroutine has been integrated directly into NEO. This provides a direct comparison between NEO and NCLASS for the same plasma parameters. A driver program was written to convert the NEO geometry and equilibrium plasma parameters into the NCLASS input for experimental profiles. The driver works for multiple ion species and general geometry. A future upgrade will also do this for local mode. A comparison between NEO and NCLASS for the poloidal flows and bootstrap current for a DIII-D L-mode discharge is shown in figure 1. Here the NEO simulations were done using the HS0 collision operator (equivalent to the NCLASS collision model) and in the weak rotation limit (since NCLASS does not include rapid toroidal rotation effects). The two codes agree qualitatively well over the entire profile. Little effect was seen from the models for potato orbits and orbit-squeezing in NCLASS for this case.

The upgrade of the NEO collision model to the full linearized FokkerPlanck operator is continuing. A monomial expansion in normalized velocity, $x_{a}=v /\left(\sqrt{2} v_{t a}\right)$, has been implemented. Here we expand the $\mathrm{n}$-th order perturbed distribution function as

$$
f_{n a}=f_{0 a} \sum_{l=0}^{N_{\xi}} \sum_{m=0}^{N_{\varepsilon}} \hat{f}_{n a}^{l m} P_{l}(\xi) x_{a}^{\alpha m+\beta l}
$$

Presently, we use $\alpha=1$ and $\beta=0$. Preliminary results with the Connor collision operator are shown in figure 2 . We find a rapid loss in accuracy (including a loss of ambipolarity) as $N_{\varepsilon}$ increases, due to round-off errors. To correct this, we instead expand the distribution function in the more 
well-behaved and bounded associated Laguerre polynomials, i.e.

$$
f_{n a}=f_{0 a} \sum_{l=0}^{N_{\xi}} \sum_{m=0}^{N_{\varepsilon}} \hat{f}_{n a}^{l m} P_{l}(\xi) L_{m}^{k(l)}\left(x_{a}^{\alpha}\right) x_{a}^{\beta l} .
$$

The integral applied to the kinetic equation to form the matrix equation thus becomes

$$
I \equiv\left(\frac{n_{0 a}}{(2 \pi)^{3 / 2} v_{t a}^{3}}\right)^{-1}\left(\frac{2 l+1}{2}\right) \int_{-1}^{1} d \xi P_{l}(\xi) \int_{0}^{\infty} d x_{a} L_{m}^{k(l)}\left(x_{a}^{\alpha}\right) x_{a}^{\beta l+2} .
$$

Presently, we take $k=0, \alpha=1, \beta=0$. (Note that $k=l+1 / 2, \alpha=2$, $\beta=1$ corresponds to the Burnett function expansion. The optimal values for these parameters is still to be determined.) In the implementation, the matrix elements in this basis are actually formed from the monomial basis elements, noting that the Laguerre polynomials can be constructed as

$$
L_{m}^{k}\left(x_{a}^{\alpha}\right)=\sum_{j=0}^{m}(-1)^{j} \frac{(m+k) !}{(m-j) !(k+j) ! j !} x^{\alpha j} .
$$

This will be essential for the full linearized operator (See our Oct report. The double integral functions which arise in the monomial basis can be computed accurately from Gamma and Beta functions.) The results, shown in figure 2, show much better convergence with $N_{\varepsilon}$. Tests for multi-species plasmas and implementation of the HS0 and full linearized Fokker-Planck collision operators are in progress. 

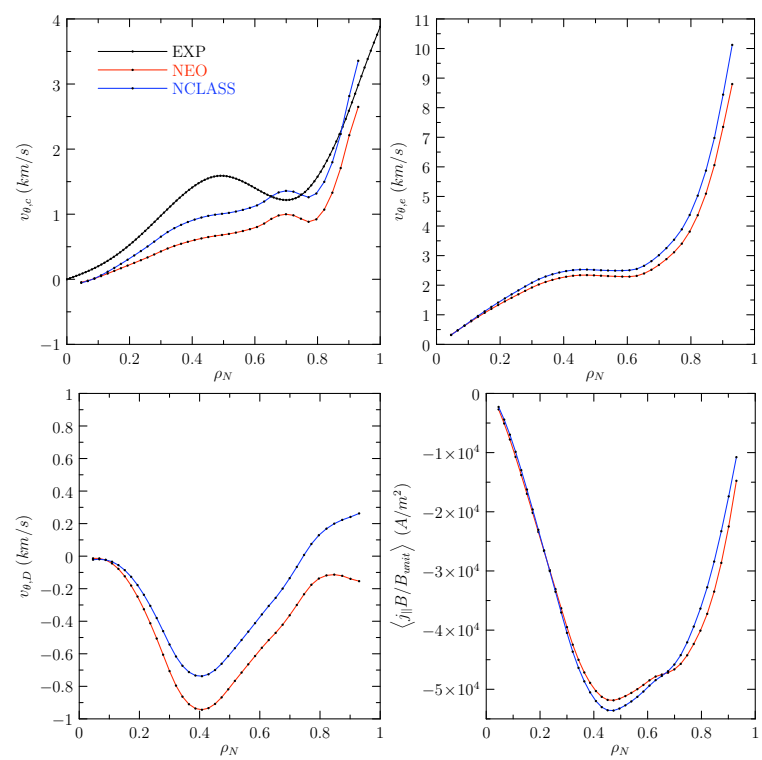

Figure 1: Radial profiles of the carbon impurity, deuterium ion, and electron neoclassical poloidal flows and the bootstrap current for DIII-D Lmode shot \#140996 comparing NEO and NCLASS simulation results. The experimentally-measured value for the carbon flow is also shown. 


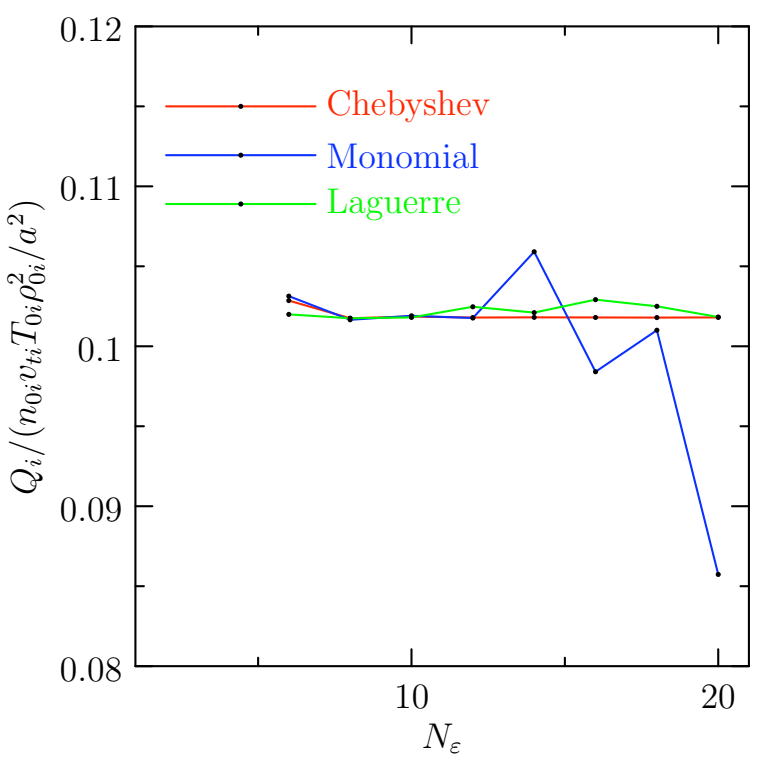

Figure 2: Convergence of the ion energy flux with number of energy polynomials comparing the old Chebyshev expansion with the new monomial and Laguerre expansions in normalized velocity in NEO. 


\title{
ESL/GA Progress Report
}

\author{
E. A. Belli, J. Candy, P. B. Snyder
}

December 2010

Our manuscript describing the new GYRO eigenvalue solver and applications to linear gyrokinetic studies of compressional magnetic field effects in NSTX-like plasmas has been published: E. Belli and J. Candy, "Fully Electromagnetic Gyrokinetic Eigenmode Analysis of High-Beta Shaped Plasmas". Phys. Plasmas, vol. 17, 112314 (2010).

For various DIII-D H-mode discharges, NEO was used to systematically study finite-orbit-width (FOW) effects due to steep gradients and access the validity of local neoclassical transport in the near-edge region. The simulations were done with two species (deuterium ions and kinetic electrons). Impurities were not included since the equilibrium profile data came from the PEQDSK file format, which presently does not include this data. Fully general geometry (including up-down asymmetry effects) was included in the simulations and the zeroth-order Hirshman-Sigmar collision operator was used. Three discharges were studied: 1) \#132010 (typical pedestal width and height), 2) \#132003 (narrow pedestal), and 3) \#132007 (large pedestal width). The equilibrium profiles and comparison of the gradientscale lengths with the gyro-radius are shown in figure 1 . We have first focused on temperature and density gradient-driven FOW effects, without orbit-squeezing effects (i.e. $E_{r}=0$ ). The results from the higher-order NEO calculations are shown in figures 2-4. A summary of the interpretation of the results is as follows:

1. No break-down of local neoclassical theory is observed for quantities which vary on the electron scale (i.e. $\left.Q_{e}\right)$

2. Local neoclassical theory appears to be valid for the bootstrap current in the near-edge. 
3. Only a moderate finite-orbit-width effect is found for the ion energy flux. Nonlinear-F may be required for $Q_{i}$ for $\psi_{N}>0.93$. But other effects not included here (such as ion orbit loss) may be more important in this region.

4. Up-down asymmetry flux-surface shaping effects on the non-local transport are weak. This is indicated by the fact that the break-down in $Q_{i}$ occurs mainly in the fourth-order component of the flux. (The thirdorder component is exactly zero for up-down symmetric plasmas.)

5. Adequate numerical resolution is essential. Specifically, high $\theta$-grid resolution is needed in the edge, due to the strong shaping effects. In fact, we find that inadequate resolution artificially enhances the FOW effect. For these simulations, we found convergence with $N \theta=31$, which is two times the standard resolution.

Results including the electric field are shown in figure 5. Here we find that there are no additional FOW effects due to $E_{r}$ in the near-edge. However, in the near-axis region, orbit-squeezing-driven effects due to potato orbits extend further into the core. 

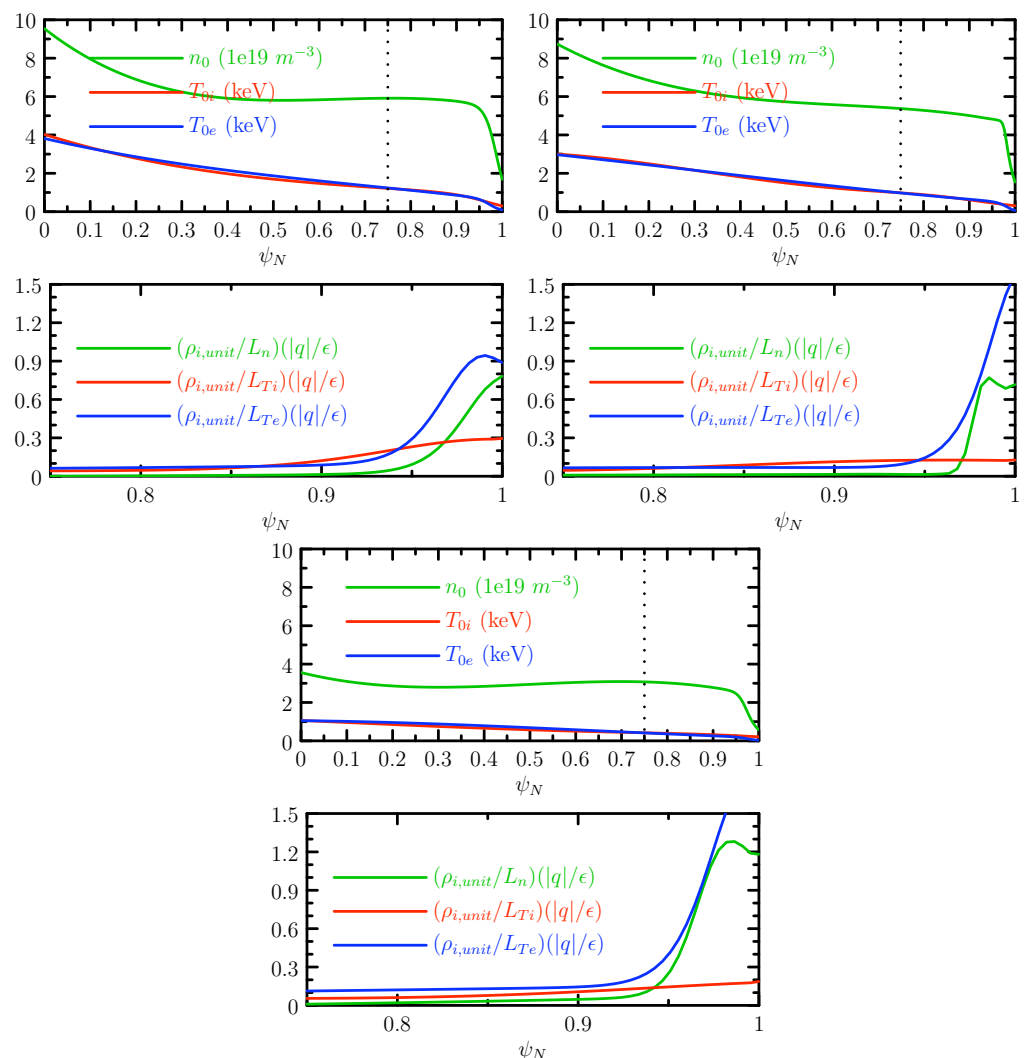

Figure 1: Profile variation of the equilibrium-scale density and ion and electron temperatures for DIII-D H-mode discharges \#132010 (top left), \#132003 (top right), and \#132007 (bottom). 

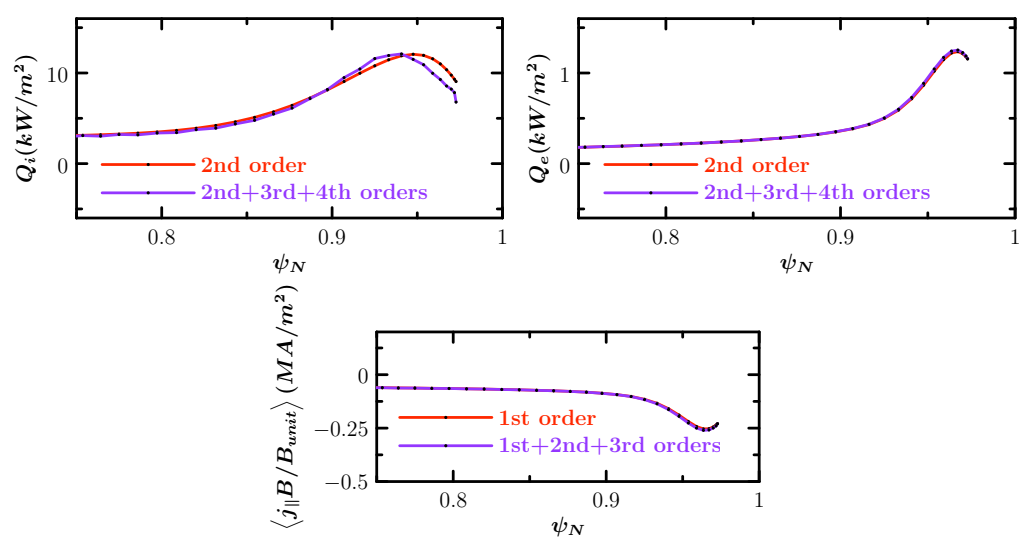

Figure 2: NEO higher-order results for the ion and electron energy fluxes and the bootstrap current for DIII-D H-mode discharge \#132010 (typical pedestal width and height case), comparing the local neoclassical results (red curves) with the total higher-order results including the non-local corrections. These simulations do not include $E_{r}$ effects.
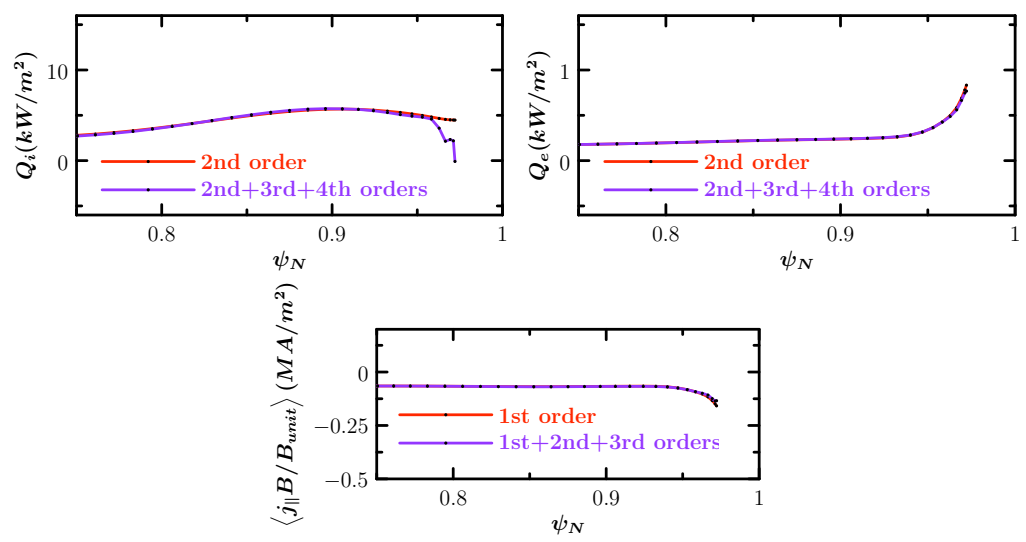

Figure 3: NEO higher-order results for the ion and electron energy fluxes and the bootstrap current for DIII-D H-mode discharge \#132003 (narrow pedestal case), comparing the local neoclassical results (red curves) with the total higher-order results including the non-local corrections. These simulations do not include $E_{r}$ effects. 

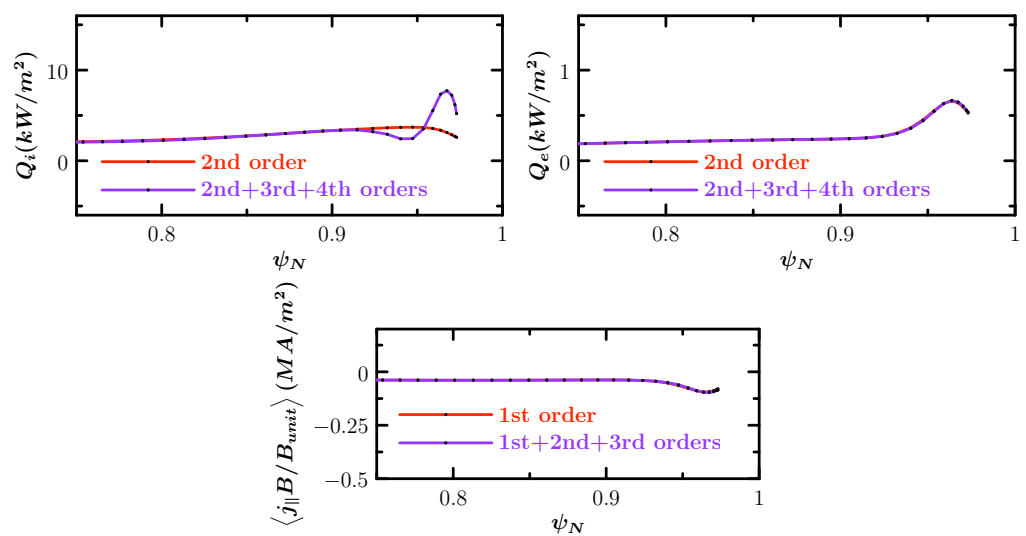

Figure 4: NEO higher-order results for the ion and electron energy fluxes and the bootstrap current for DIII-D H-mode discharge \#132007 (large pedestal width case), comparing the local neoclassical results (red curves) with the total higher-order results including the non-local corrections. These simulations do not include $E_{r}$ effects.
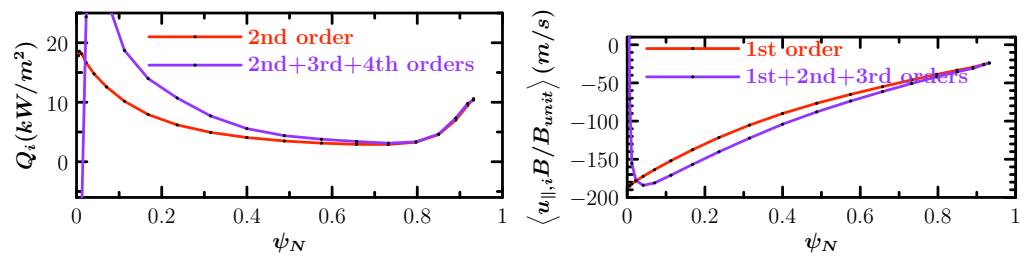

Figure 5: NEO higher-order results for the ion energy fluxe and ion parallel flow for DIII-D H-mode discharge \#132010, including the radial electric field effects, comparing the local neoclassical results (red curves) with the total higher-order results including the non-local corrections. These simulations do not include kinetic electrons. 


\title{
ESL/GA Progress Report
}

\author{
E. A. Belli, J. Candy, P. B. Snyder
}

January 2011

Work has continued on the implementation of the full linearized FokkerPlanck collision operator in NEO. A pure monomial basis function expansion in normalized velocity $\left(x_{a}=v /\left(\sqrt{2} v_{t a}\right)\right.$, i.e.

$$
f_{1 a}=f_{0 a} \sum_{l=0}^{N_{\xi}} \sum_{m=0}^{N_{\varepsilon}} \hat{f}_{1 a}^{l m} P_{l}(\xi) x_{a}^{2 m+\beta l},
$$

unsurprisingly leads to an ill-conditioned matrix. Thus, variations of a Laguerre expansion in energy of the form

$$
f_{1 a}=f_{0 a} \sum_{l=0}^{N_{\xi}} \sum_{m=0}^{N_{\varepsilon}} \hat{f}_{1 a}^{l m} P_{l}(\xi) L_{m}^{k(l)}\left(x_{a}^{2}\right) x_{a}^{\beta l},
$$

were explored, constructing the matrix elements from the monomial moments as described in our previous report. A simple test case with a single ion species and adiabatic electrons is used and comparisons are made with our previous implementation of the HS0 collision operator using a Chebyshev expansion in re-normalized velocity (see figure 1). As shown in figure 2 , we have found that the Sonine expansion $(k(l)=l+1 / 2, \beta=1)$ has issues with numerical accuracy, specifically with numerical precision loss for the high-power elements due to the $x_{a}^{\beta l}$ factor. This was confirmed by comparing the matrix elements computed using standard double precision with those computed using quad precision.

A traditional Laguerre expansion $(k(l)=1 / 2, \beta=0)$, which we call "Laguerre-0", is also not ideal. The convergence results for this method are shown oin figure 3 . While the convergence plots look smoother than the 
Sonine convergence plots (indicating that this method does not appear to have the same precision loss problem, at least not up to $\mathrm{N}=20$ ), even at large collision frequency the convergence is very slow, particularly for $u_{\|}$ and, in accordance, the ambipolarity is not good. This is most likely due to the fact this method is really expanding $f$ in powers of $\varepsilon$, so the odd-l Legendre components of $f$ are slow to converge because it has to use a lot of polynomials to make functions that look like half-powers of energy. It also lacks the extra $x^{l}$ factor in the $f$-expansion which force the higher-powers in $f$ to go to zero more quickly.

To test this, we have compared these results with a "Laguerre-1" method (i.e. $k(l)=3 / 2, \beta l \doteq 1$ ), as shown in figure 4 . Here we find that the energy flux convergence is nearly the same at small $\tau_{i i}^{-1}$, but the $u_{\|}$convergence is much better and the ambipolarity is good. However, the results also show that, at large collision frequency, the energy flux convergence is very slow and appears wrong even at $\mathrm{N}=20$. This is most likely due to the fact that the $l=0$ component of $f$ does not really go the zero as $x \rightarrow 0$ (it should go to a constant), so this method does not represent this component accurately.

The optimal expansion seems to be a combination of the two Laguerre methods, i.e.

$$
f_{1 a}=f_{0 a} \sum_{m=0}^{N_{\varepsilon}}\left[\hat{f}_{1 a}^{0 m} L_{m}^{1 / 2}\left(x_{a}^{2}\right)+\sum_{l=1}^{N_{\xi}} P_{l}(\xi) \hat{f}_{1 a}^{l m} L_{m}^{3 / 2}\left(x_{a}^{2}\right) x_{a}\right] .
$$

(A similar method using $L_{0}^{1 / 2}$ for $m=0, x L_{1}^{3 / 2}$ for $m=1$, and $x^{2} L_{m}^{5 / 2}$ for $m>1$ was also tried and gave similar, though slightly worse results at low collision frequency.) These results are shown in figure 5. The method looks as good as the old Chebyshev method with the less-complicated HS0 operator.

The next step is to optimize the method for inclusion of kinetic electrons. Preliminary results find that numerical accuracy and overflow/underflow issues arise, even at relatively small values of $N$, for the cross-species field particle component of the operator. 

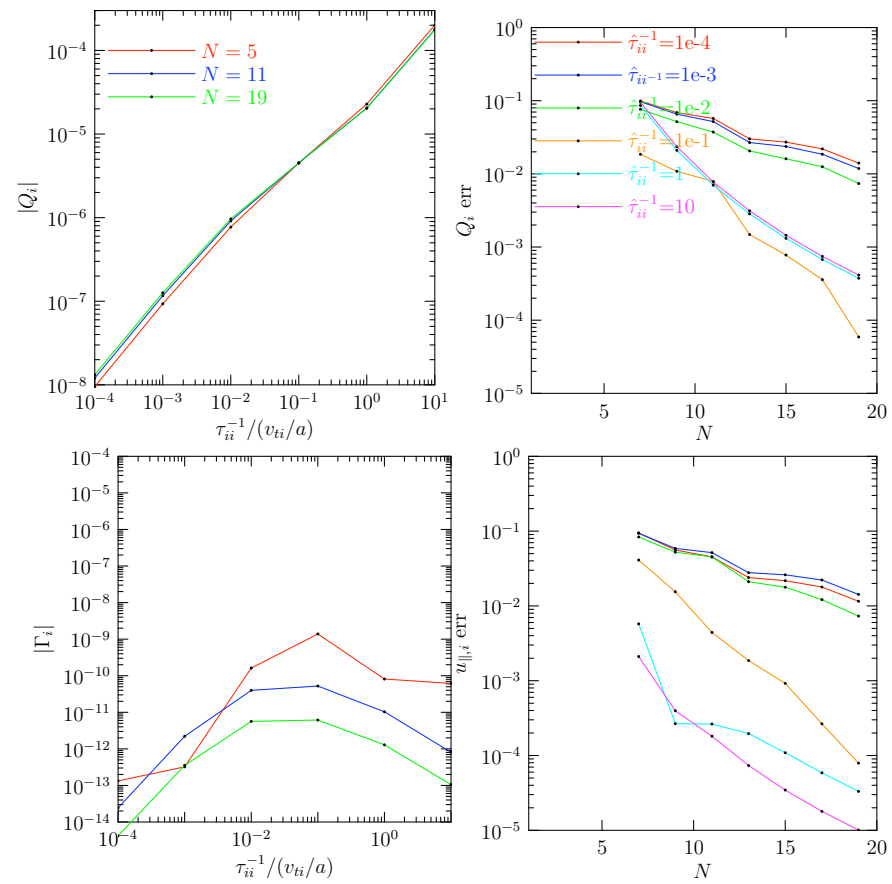

Figure 1: (left) Variation of the ion energy flux and ion particle flux with collision frequency for the case of a single ion species and adiabatic electrons at various number of grid points $N=N_{\theta}=N_{\xi}=N_{\varepsilon}$. (right) Convergence of the ion energy flux and ion parallel flow with $N$ at various collision frequencies. These results used the HS0 collision operator and a Chebyshev expansion in re-normalized velocity. 

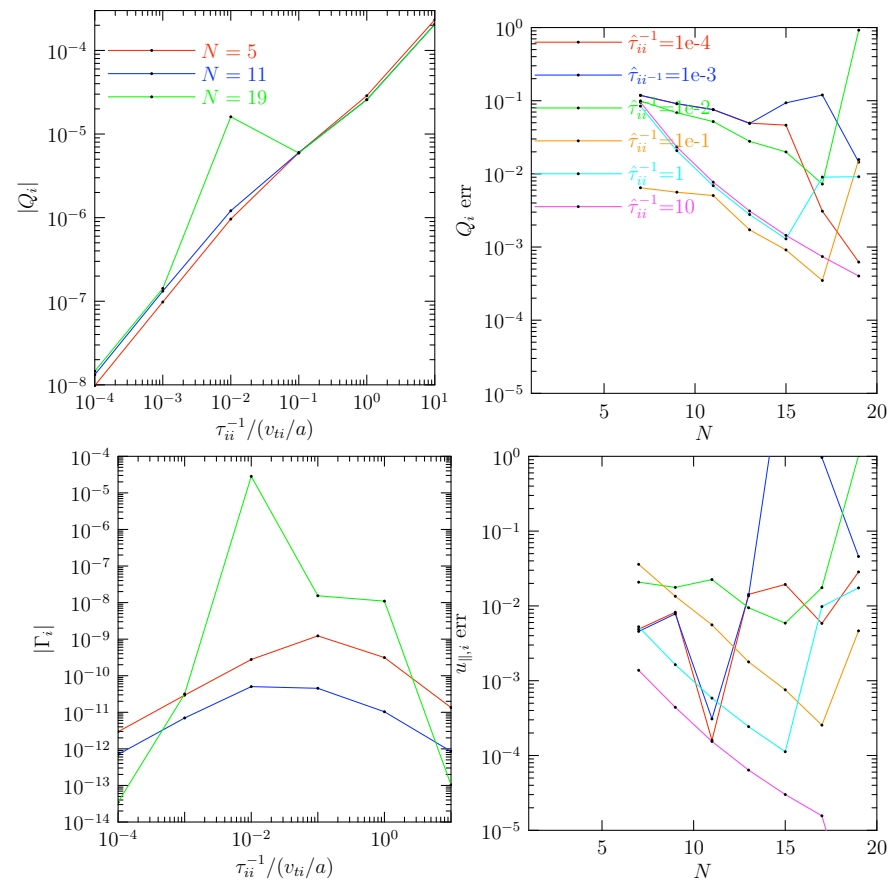

Figure 2: (left) Variation of the ion energy flux and ion particle flux with collision frequency for the case of a single ion species and adiabatic electrons at various number of grid points $N=N_{\theta}=N_{\xi}=N_{\varepsilon}$. (right) Convergence of the ion energy flux and ion parallel flow with $N$ at various collision frequencies. These results used the full linearized FP collision operator and a Sonine expansion in normalized energy. 

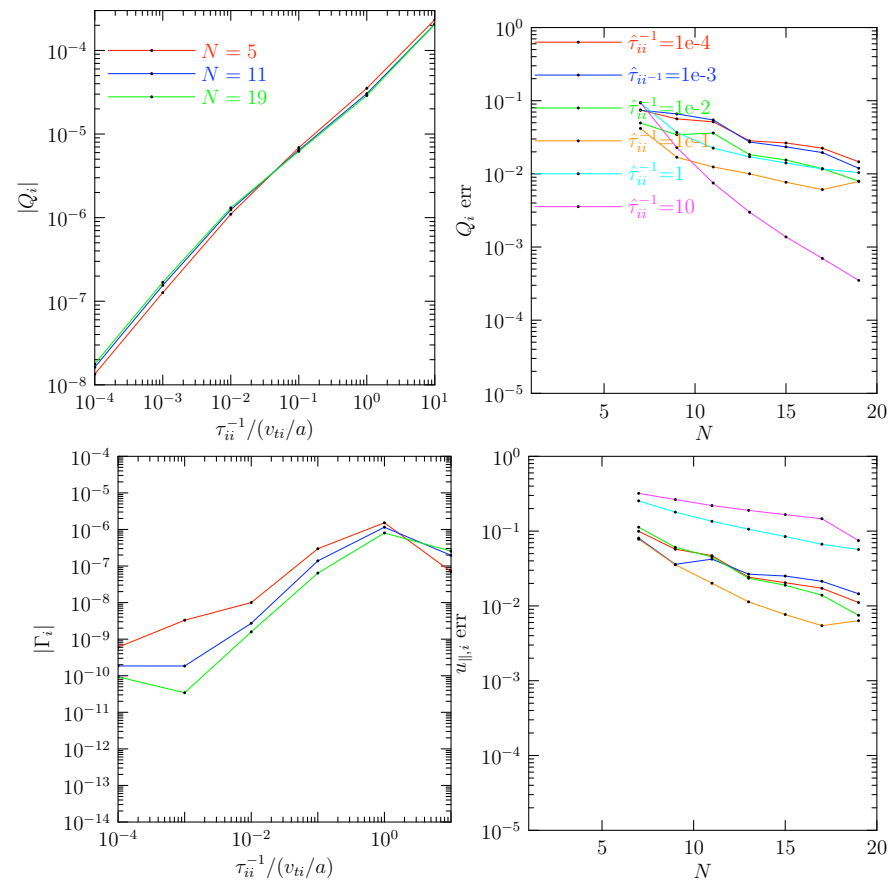

Figure 3: (left) Variation of the ion energy flux and ion particle flux with collision frequency for the case of a single ion species and adiabatic electrons at various number of grid points $N=N_{\theta}=N_{\xi}=N_{\varepsilon}$. (right) Convergence of the ion energy flux and ion parallel flow with $N$ at various collision frequencies. These results used the full linearized FP collision operator and a Laguerre-0 expansion in normalized energy. 

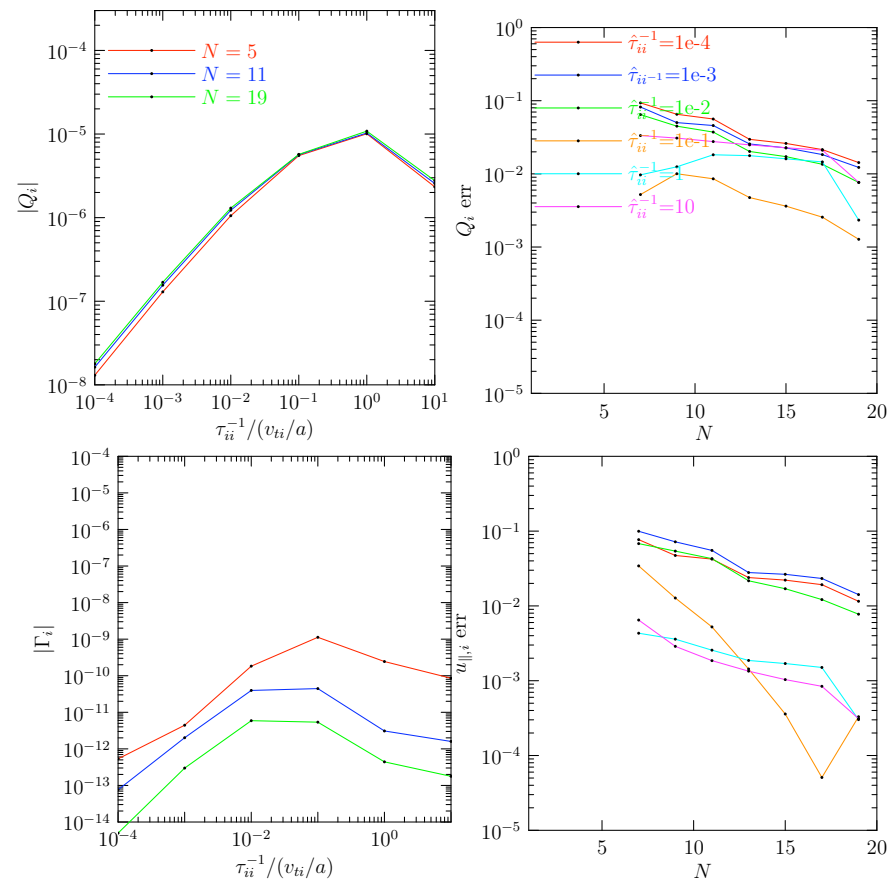

Figure 4: (left) Variation of the ion energy flux and ion particle flux with collision frequency for the case of a single ion species and adiabatic electrons at various number of grid points $N=N_{\theta}=N_{\xi}=N_{\varepsilon}$. (right) Convergence of the ion energy flux and ion parallel flow with $N$ at various collision frequencies. These results used the full linearized FP collision operator and a Laguerre-1 expansion in normalized energy. 